\title{
THE EXIT PATH OF A MARKOV CHAIN WITH RARE TRANSITIONS
}

\author{
OLIVIER CATONI AND RAPHAËL CERF
}

\begin{abstract}
We study the exit path from a general domain after the last visit to a set of a Markov chain with rare transitions. We prove several large deviation principles for the law of the succession of the cycles visited by the process (the cycle path), the succession of the saddle points gone through to jump from cycle to cycle on the cycle path (the saddle path) and the succession of all the points gone through (the exit path). We estimate the time the process spends in each cycle of the cycle path and how it decomposes into the time spent in each point of the exit path. We describe a systematic method to find the most likely saddle paths. We apply these results to the reversible case of the Metropolis dynamics. We give in appendix the corresponding large deviation estimates in the non homogeneous case, which are corollaries of already published works by Catoni (1992) and Trouvé $(1992,1996 a)$.
\end{abstract}

\section{INTRODUCTION}

Markov chains with rare transitions appear in a variety of contexts ranging from statistical mechanics to optimization and reliability theory. This discrete model of a dynamical system with small random perturbations has been studied for itself for the first time by Freidlin and Wentzell in connection with diffusion models (1984). Its main feature is that the transition rates are supposed to obey some large deviation principle with parameter $\beta$ (called the inverse temperature). In other words the transition rate between states $x$ and $y$ is assumed to be of order $\exp -\beta V(x, y)$ for some rate function $V$. Exponential rates come from the discrete approximation of more complex events, such as the jumps of a diffusion process with small perturbations from one attractor to another, or the rate of failure of a machine. They may also be used to simulate on a computer the distribution at thermal equilibrium of a system of particles or magnetic spins, called the Gibbs ensemble by physicists. They may even be used as a rough model for the microscopic dynamics of such a system of statistical mechanics (whether at equilibrium or not).

The convergence of Markov chains with rare transitions towards equilibrium becomes arbitrarily slow when temperature goes to zero. To solve this problem, which is crucial for simulations and optimization applications, time inhomogeneous Markov chains have been introduced under the name

ESAIM: Probability and Statistics is an electronic journal with URL address http://www.emath.fr/ps/

Received by the journal October 17, 1995. Revised July 4, 1996. Accepted for publication October 10, 1996.

(c) Société de Mathématiques Appliquées et Industrielles.Typeset by $\mathcal{A} \mathcal{M} S$-TEX. 
of simulated annealing algorithms. Many questions have been studied about the low temperature behaviour of both homogeneous and non homogeneous chains. Let us mention three of them: the question of the limiting behaviour of their invariant distributions (which is somehow a prerequisite to any further study), the rate of convergence of these chains towards equilibrium at low temperatures and the distribution of the exit times and points from subdomains of the state space. Two main approaches have proved successful to answer these questions: the semi-group approach, based on spectral gap estimates, and large deviation theory, where the distributions of exit times and points are estimated first. The semi-group approach started with Holley and Stroock (1988) (see also Diaconis and Stroock (1991)) and was first applied globally, to get results on the rate of convergence of simulated annealing algorithms toward equilibrium for slow evolutions of the temperature (see also Miclo (1991)). Then it was realized that it was possible to localize it to get results on exit times and exit points. The first step in this direction was made by Götze (1991). It led to precise estimates for the joint distribution of the exit point and exit time from arbitrary subdomains and (almost) arbitrary $C^{1}$ temperature evolutions in Miclo $(1995,1996)$, in case of continuous time dynamics. For a different point of view, based on backward equations and requiring some restrictive assumptions on the rate of decay of the temperature, see also Chiang and Chow (1995).

The large deviation approach started with Freidlin and Wentzell's book on "Random Perturbations of Dynamical Systems", where the homogeneous case is studied by graph techniques. Freidlin and Wentzell's theory gives closed formulas for the distribution of the exit point from arbitrary subdomains and the expectation of the corresponding exit time. These authors have also introduced a fundamental concept in the study of trajectories: the decomposition of the state space into cycles. A cycle is a subdomain in which the chain stays an exponential length of time and goes to and fro an exponential number of times between any pair of states before leaving. The construction of the cycles in the most general situation was not given by Freidlin and Wentzell, and was described in full detail for the first time seemingly by Hwang and Sheu (acknowledged in Chiang and Chow (1989), and published in (1992), but the preprint circulated a long time before). The study of the exit time and point from subdomains in the non homogeneous case was made in Catoni $(1990,1991 \mathrm{a}, 1992)$ for arbitrary non increasing temperature evolutions and reversible simulated annealing algorithms. The rough estimates (where the exponents are optimal but the constants before the exponentials are not) were generalized to non necessarily reversible chains and arbitrary non increasing temperature evolutions by Trouvé (1992, 1996a) (English translations (1996a, 1996b)). In these papers, the line of reasoning is inspired by Freidlin and Wentzell's theory and the graph technique is replaced by induction proofs. Applications are made to the study of optimal or nearly optimal temperature evolutions (those giving approximately the largest probability to be in a state below a given energy level after some fixed large number of iterations). These tools were also applied to the theory of genetic algorithms by Cerf $(1994,1996 \mathrm{a})$. The first main question addressed in this context is the asymptotic behaviour of the in- 
variant distribution (1993). Further insights into the dynamics of genetic algorithms can be obtained by analyzing the influence of the population size and the operators (mutation, crossover, selection) on the geometry of the cycles $(1996 \mathrm{~b}, 1996 \mathrm{c})$.

The large deviation approach was the first one to give results on the behaviour of trajectories and also the first one to be generalized to arbitrary non increasing temperature evolutions, this extension being made in the case of discrete time Markov chains. Moreover the "rough large deviation estimates" given in Catoni (1992) and Trouvé (1996a) have the advantage to be uniform with respect to the energy function (or with respect to the rate function in the non reversible case). This allowed to prove that some type of exponential triangular temperature evolutions are robust, in the sense that they give an almost optimal convergence rate uniformly over compact sets of energy (or rate) functions.

Recently an important step was made in Miclo $(1995,1996)$ along the semi-group approach. These two papers are the first from the "semigroup school" to deal with arbitrary $C^{1}$ temperature evolutions in the nonreversible case. They study the continuous time case, under the assumption that the generator is exactly equal to $q(x, y) \exp -\beta_{t} V(x, y)$ (whereas in Trouvé (1996a) the transition matrix has only to be of the same order as this quantity), and prove results for the renormalized exit times and points from subdomains with "sharp" constants. Sharp constants were also obtained in Catoni (1991a) for reversible Markov chains, and the generalization to the non reversible case should be feasible, but would be more complicated than Miclo's proof, which does not rely on an induction argument.

Freidlin and Wentzell's theory also had an echo in the domain of statistical physics, where homogeneous Markov chains with rare transitions were studied in connection with the metastability phenomenon (Olivieri and Scoppola $(1995,1996))$. In the study of metastability, one is interested in the occurrence of a partial equilibrium in a subdomain of the state space, as well as in the lifetime of these partial equilibria. The global relaxation time of the system is the time needed for partial equilibria to melt into a global one. The cycle decomposition of Freidlin and Wentzell gives a clear cut description of the metastability phenomenon and was reworked in this context by Olivieri and Scoppola $(1995,1996)$. One of the merits of these papers is that they have underlined the importance of the description of the escape path from metastable states for the understanding of the metastability phenomenon. They give a description of the most probable escape paths and a physical interpretation of the cycle decomposition in terms of renormalization procedures (see also Scoppola (1993)).

The aim of this paper is to give a large deviation description of the escape path in the homogeneous case, using only simple proofs inherited directly from Freidlin and Wentzell's graph method. We will prove various large deviation estimates related to the exit path of a homogeneous Markov chain with rare transitions from an arbitrary subdomain of the state space. Doing this, we will have in view mainly applications to the metastability problem, which will be given in Alonso and Cerf (1996) and in Ben Arous and Cerf (1996). 
Let us also mention that, as proved in Catoni (1996) and Cot and Catoni (1996), piecewise constant temperature evolutions, if properly tuned, can give almost optimal convergence rates for the generalized simulated annealing algorithm. Moreover, another speed-up technique for the Metropolis algorithm, called the Iterated Energy Transformation algorithm and studied in Catoni (1994), uses homogeneous Markov chains with rare transitions. Therefore the homogeneous case is also relevant for stochastic optimization.

We could have started with refined estimates from which we would have deduced coarser and coarser ones using the contraction principle of large deviation theory. We have instead chosen to proceed step by step from simple estimates to more refined ones. Although this is not the most economical approach from the mathematical point of view, we think it gives an easier understanding of the behaviour of the process. We start with a rough description of the exit path, where we study only the succession of the maximal cycles the process goes through. Then we look for the entrance and exit points of the visited cycles, thus describing the exit saddle path. We give precise estimates for the time the process spends in each cycle of the cycle path. We describe eventually more precisely the succession of arrows the trajectory is likely to go through and we give estimates for the time spent in each point. We put forward an efficient method to find the exit saddle paths the system is likely to take. We also simplify some results in the reversible case of a Metropolis dynamics. This method is applied to study the metastability of the three dimensional Ising model on a torus at very low temperatures in Ben Arous and Cerf (1996), the model dependent variational problems being solved in Alonso and Cerf (1996). This study of a huge and intricate energy landscape would not have been possible without the use of a systematic way to find the exit path and illustrates the efficiency of the method.

We had claimed in our first submitted manuscript that we would basically prove nothing new if compared with the non homogeneous and therefore more general results contained in Catoni (1992) and in Trouvé (1996a). As one of the referees suggested to make this statement more precise, we give in appendix the non homogeneous formulation of the main results of the paper and indicate how to deduce them from Trouvé (1996a) (English translation Trouvé (1996b)).

\section{THE MAIN PROBLEM}

Let $E$ be a finite space. We consider a family of time homogeneous Markov chains $\left(X, P_{\beta}\right)$ on $E$ indexed by a positive parameter $\beta$ (the inverse temperature). More precisely, we consider the coordinate process $X=\left(X_{n}\right)_{n \in \mathbb{N}}$ on the space $E^{\mathbb{N}}$ defined by $X_{n}:\left(\omega_{0}, \ldots\right) \mapsto \omega_{n}$ together with a family of probabilities $\left(P_{\beta}\right)$ indexed by $\beta$; under each of them the coordinate process is a Markov chain. We suppose that these Markov chains are in the Freidlin-Wentzell regime, namely that their transition mechanisms satisfy

$$
a(\beta) \exp -\beta V(x, y) \leq P_{\beta}\left(X_{n+1}=y / X_{n}=x\right) \leq a(\beta)^{-1} \exp -\beta V(x, y)
$$


for all $x, y$ in $E$, where $\beta \mapsto a(\beta)$ is a positive function such that

$$
\lim _{\beta \rightarrow \infty} \beta^{-1} \ln a(\beta)=0
$$

and $V: E \times E \rightarrow \mathbb{R}_{+} \cup\{\infty\}$ is an irreducible cost function i.e.

$$
\begin{aligned}
\forall x, y \in E \times E \quad \exists i_{0}, i_{1}, \ldots, i_{r} \quad i_{0}= & x, \quad i_{r}=y, \\
& V\left(i_{0}, i_{1}\right)+\cdots+V\left(i_{r-1}, i_{r}\right)<\infty .
\end{aligned}
$$

For $C$ an arbitrary subset of $E$ we define the time $\tau(C, m)$ of exit from $C$ after time $m$

$$
\tau(C, m)=\min \left\{n \geq m: X_{n} \notin C\right\}
$$

(we make the convention that $\tau(C)=\tau(C, 0)$ ).

We define also the time $\theta(C, m)$ of the last visit to the set $C$ before time $m$

$$
\theta(C, m)=\max \left\{n \leq m: X_{n} \in C\right\}
$$

(if the chain has not visited $C$ before $m$, we take $\theta(C, m)=0$ ).

Remark that $\tau$ is a stopping time when $m$ is deterministic whereas $\theta$ isn't.

Let $G$ and $D$ be two subsets of $E$ such that $G \subset D$ and let $x$ be a starting point in $G$. Our aim is to describe the behaviour of the chain $\left(X_{n}\right)$ after its last visit to the set $G$ before it escapes from the set $D$. More precisely we will study the asymptotic behaviour as $\beta$ goes to infinity of the law of $\left(X_{k}, \theta(G, \tau(D)) \leq k \leq \tau(D)\right)$. We will determine the points of $D \backslash G$ the Markov chain $\left(X_{n}\right)$ is likely to visit on its exit path as well as the typical times it spends in the subsets of $D \backslash G$ it crosses. The key idea to achieve this study, introduced by Catoni (1991a), is to decompose $D \backslash G$ into its maximal cycles and to focus on the jumps of $\left(X_{n}\right)$ between these cycles, which may be seen as abstract states.

\section{Freidlin and Wentzell lemmas on Markov Chains}

These lemmas give useful formulas for the invariant measure and for the laws of the exit time and exit point for an arbitrary subset of $E$. These formulas are rational fractions of the coefficients of the transition matrix whose numerators and denominators are most conveniently written as sums over particular types of graphs.

Definition 3.1. (the graphs $G(W)$ ) Let $W$ be an arbitrary non-empty subset of $E$.

An oriented graph on $E$ is called a $W$-graph if and only if

- there is no arrow starting from a point of $W$;

- each point of $W^{c}$ is the initial point of exactly one arrow;

- for each point $x$ in $W^{c}$, there exists a path in the graph leading from $x$ to $W$.

The set of all $W$-graphs is denoted by $G(W)$.

REMARK. The third condition above is equivalent to

- there is no cycle in the graph. 
Definition 3.2. (the graphs $G_{x, y}(W)$ ) Let $W$ be an arbitrary non-empty subset of $E$, let $x$ belong to $E$ and $y$ to $W$.

If $x$ belongs to $W^{c}$, the set $G_{x, y}(W)$ is the set of all oriented graphs on $E$ such that

- there is no arrow starting from a point of $W$;

- each point of $W^{c}$ is the initial point of exactly one arrow;

- for each point $z$ in $W^{c}$, there exists a path in the graph leading from $z$ to $W$

- there exists a path in the graph leading from $x$ to $y$.

More concisely, they are the graphs of $G(W)$ which contain a path leading from $x$ to $y$.

If $x$ belongs to $W$, the set $G_{x, y}(W)$ is empty if $x \neq y$ and is equal to $G(W)$ if $x=y$.

Remark. The graphs in $G_{x, y}(W)$ have no cycles. For any $x$ in $E$ and $y$ in $W$, the set $G_{x, y}(W)$ is included in $G(W)$.

DEFINITION 3.3. (the graphs $G(x \not \rightarrow W)$ ) Let $W$ be an arbitrary non-empty subset of $E$ and let $x$ be a point of $E$.

If $x$ belongs to $W$ the set $G(x \nrightarrow \rightarrow W)$ is empty.

If $x$ belongs to $W^{c}$ the set $G(x \nrightarrow W)$ is the set of all oriented graphs on $E$ such that

- there is no arrow starting from a point of $W$;

- each point of $W^{c}$ except one, say $y$, is the initial point of exactly one arrow;

- there is no cycle in the graph;

- there is no path in the graph leading from $x$ to $W$.

The third condition (no cycle) is equivalent to

- for each $z$ in $W^{c} \backslash\{y\}$, there is a path in the graph leading from $z$ to $W \cup\{y\}$.

Lemma 3.4. Let $W$ be an arbitrary non-empty subset of $E$ and let $x$ be a point of $E$.

The set $G(x \nrightarrow \rightarrow)$ is the union of all the sets $G_{x, y}(W \cup\{y\}), y \in W^{c}$.

REMARK. In the case $x \in W^{c}, y \in W$, the definitions of $G_{x, y}(W)$ and $G(x \not t$ $W)$ are those given by Wentzell and Freidlin (1984). We have extended these definitions to cover all possible values of $x$. With our choice for the definition of the time of exit $\tau\left(W^{c}\right)$ (the first time greater than or equal to zero when the chain is outside $\left.W^{c}\right)$, the formulas for the law of $X_{\tau\left(W^{c}\right)}$ and for the expectation of $\tau\left(W^{c}\right)$ will remain valid in all cases.

Let $g$ be a graph on $E$, we define its probability $p_{\beta}(g)$ by

$$
p_{\beta}(g)=\prod_{(x \rightarrow y) \in g} P_{\beta}\left(X_{n+1}=y / X_{n}=x\right)
$$


LEMma 3.5. (expected number of visits before exit) For any non-empty subset $W$ of $E, y$ in $W^{c}$ and $x$ in $E$,

$$
\sum_{n=0}^{\infty} P_{\beta}\left(X_{n}=y, \tau\left(W^{c}\right)>n / X_{0}=x\right)=\frac{\sum_{g \in G_{x, y}(W \cup\{y\})} p_{\beta}(g)}{\sum_{g \in G(W)} p_{\beta}(g)} .
$$

This lemma, due to Catoni (1996), may be used to prove the following three lemmas of Wentzell and Freidlin. For the sake of completeness, we reproduce the proof here.

Proof. Let us dismiss in this proof the subscript $\beta$, the lemma being true for any irreducible Markov chain with transition matrix $p$. The matrix

$$
m(x, y)=\sum_{n=0}^{\infty} P\left(X_{n}=y, \tau\left(W^{c}\right)>n / X_{0}=x\right)
$$

is the unique solution of

$$
\sum_{z \in W^{c}}(I(x, z)-p(x, z)) m(z, y)=I(x, y), \quad x, y \in W^{c},
$$

because $(I-p)_{\mid W^{c} \times W^{c}}$ is invertible ( $p$ being irreducible). As

$$
p(x, x)=1-\sum_{z \in E \backslash\{x\}} p(x, z),
$$

the preceding equation can be rewritten as

$$
\sum_{z \in E \backslash\{x\}} p(x, z) m(x, y)=I(x, y)+\sum_{z \in(W \cup\{x\})^{c}} p(x, z) m(z, y) .
$$

Let

$$
\tilde{m}(x, y)=\sum_{g \in G_{x, y}(W \cup\{y\})} p(y)\left(\sum_{g \in G(W)} p(g)\right)^{-1}
$$

We have

$$
\sum_{z \in\{x\}^{c}} p(x, z) \tilde{m}(x, y)=\left(\sum_{(z, g) \in C_{1}} p(x, z) p(g)\right)\left(\sum_{g \in G(W)} p(g)\right)^{-1},
$$

and

$$
\sum_{z \in(W \cup\{x\})^{c}} p(x, z) \widetilde{m}(z, y)=\left(\sum_{(z, g) \in C_{2}} p(x, z) p(g)\right)\left(\sum_{g \in G(W)} p(g)\right)^{-1}
$$

where

$$
\begin{aligned}
& C_{1}=\left\{(z, g) \in E \backslash\{x\} \times G(W \cup\{y\}): g \in G_{x, y}(W \cup\{y\})\right\}, \\
& C_{2}=\left\{(z, g) \in E \backslash(W \cup\{x\}) \times G(W \cup\{y\}): g \in G_{z, y}(W \cup\{y\})\right\} .
\end{aligned}
$$


For a graph $g$ with exactly one arrow starting from $x$, we denote by $g(x)$ the unique element $z$ such that the arrow $x \rightarrow z$ is present in $g$. In the case when $x \neq y$, consider the one to one change of variable $\varphi: C_{1} \rightarrow C_{2}$ defined by

$$
\varphi(z, g)=\left\{\begin{array}{cl}
(z, g) & \text { if } g \in G_{z, y}(W \cup\{y\}) \\
(g(x), g \cup\{(x \rightarrow z)\} \backslash\{(x \rightarrow g(x))\}) & \text { if } g \notin G_{z, y}(W \cup\{y\})
\end{array}\right.
$$

To check that it is one to one, it is enough to notice that its inverse is given by

$$
\varphi^{-1}(z, g)=\left\{\begin{array}{cl}
(z, g) & \text { if } g \in G_{x, y}(W \cup\{y\}) \\
(g(x), g \cup\{(x \rightarrow z)\} \backslash\{(x \rightarrow g(x))\}) & \text { if } g \notin G_{x, y}(W \cup\{y\})
\end{array}\right.
$$

Let $(z, g)$ belong to $C_{1}$ and let $\left(z^{\prime}, g^{\prime}\right)=\varphi(z, g)$. We check that $p(x, z) p(g)=$ $p\left(x, z^{\prime}\right) p\left(g^{\prime}\right)$.

It follows that

$$
\sum_{(z, g) \in C_{1}} p(x, z) p(g)=\sum_{\left(z^{\prime}, g^{\prime}\right) \in C_{2}} p\left(x, z^{\prime}\right) p\left(g^{\prime}\right)
$$

and thus $\tilde{m}$ satisfies equation (3.1) when $x \neq y$.

In the case when $x=y$, we have $C_{2} \subset C_{1}$, and we can consider the change of variable $\varphi: C_{1} \backslash C_{2} \rightarrow G(W)$ defined by $\varphi(z, g)=g \cup\{(y \rightarrow z)\}$. It is one to one since its inverse is given by $\varphi^{-1}(g)=(g(y), g \backslash\{(y \rightarrow g(y))\})$. Therefore

$$
\sum_{(z, g) \in C_{1} \backslash C_{2}} p(y, z) p(g)=\sum_{g \in G(W)} p(g)
$$

and

$$
\begin{aligned}
\left(\sum_{(z, g) \in C_{1}} p(y, z) p(g)\right)\left(\sum_{g \in G(W)} p(g)\right)^{-1} & = \\
& 1+\left(\sum_{(z, g) \in C_{2}} p(y, z) p(g)\right)\left(\sum_{g \in G(W)} p(g)\right)^{-1} .
\end{aligned}
$$

This shows that $\tilde{m}$ satisfies equation (3.1) also when $x=y$.

LEMMA 3.6. (stationary measure) The stationary measure $\mu_{\beta}$ of the Markov chain $\left(\left(X_{n}\right)_{n \in \mathbb{N}}, P_{\beta}\right)$ is

$$
\forall x \in E \quad \mu_{\beta}(x)=\frac{\sum_{g \in G(x)} p_{\beta}(g)}{\sum_{y \in E} \sum_{g \in G(y)} p_{\beta}(g)} .
$$

Lemma 3.7. (exit point) For any non-empty subset $W$ of $E$, any $y$ in $W$ and $x$ in $E$,

$$
P_{\beta}\left(X_{\tau\left(W^{c}\right)}=y / X_{0}=x\right)=\frac{\sum_{g \in G_{x, y}(W)} p_{\beta}(g)}{\sum_{g \in G(W)} p_{\beta}(g)} .
$$


Lemma 3.8. (exit time) For any subset $W$ of $E$ and $x$ in $E$,

$$
E_{\beta}\left(\tau\left(W^{c}\right) / X_{0}=x\right)=\frac{\sum_{y \in W^{c}} \sum_{g \in G_{x, y}(W \cup\{y\})} p_{\beta}(g)}{\sum_{g \in G(W)} p_{\beta}(g)}=\frac{\sum_{g \in G(x \nrightarrow W)} p_{\beta}(g)}{\sum_{g \in G(W)} p_{\beta}(g)} .
$$

\section{THE CYCLE DECOMPOSITION}

We now recall briefly some basic facts and definitions of quantities concerning the decomposition of $E$ into cycles. For a detailed exposition of this question, we refer the reader to Catoni $(1992,1996)$ and Trouvé $(1992,1993$, 1996a, 1996b).

Definition 4.1. (cost of a graph) We define the cost of a graph $g$ over $E$ by

$$
V(g)=\sum_{(x \rightarrow y) \in g} V(x, y)
$$

Clearly, we have $\lim _{\beta \rightarrow \infty} \ln p_{\beta}(g) / \beta=-V(g)$.

We next define a delicate but extremely useful tool.

Definition 4.2. (renormalized communication cost) Lemma 3.7 implies the existence of the limits for any subset $D$ of $E$

$$
\begin{aligned}
\forall x \in D \quad \forall y \notin D & \quad \lim _{\beta \rightarrow \infty}-\frac{1}{\beta} \ln P_{\beta}\left(X_{\tau(D)}=y / X_{0}=x\right)=C_{D}(x, y) \\
= & \min \left\{V(g): g \in G_{x, y}\left(D^{c}\right)\right\}-\min \left\{V(g): g \in G\left(D^{c}\right)\right\},
\end{aligned}
$$

$\forall x \notin D \quad \forall y \notin D \quad \lim _{\beta \rightarrow \infty}-\frac{1}{\beta} \ln P_{\beta}\left(X_{\tau(D, 1)}=y / X_{0}=x\right)=C_{D}(x, y)=$ $\min \left\{V(x, z)+V(g): z \in D \cup\{y\}, g \in G_{z, y}\left(D^{c}\right)\right\}-\min \left\{V(g): g \in G\left(D^{c}\right)\right\}$.

For $x$ in $E$ and $y$ in $D, x \neq y$, we set $C_{D}(x, y)=C_{D \backslash\{y\}}(x, y)$. For $x=y$, we set $C_{D}(x, x)=0$. Thus $C_{D}$ is defined over $E \times E$. The quantity $C_{D}(x, y)$ is called the renormalized communication cost in $D$.

For a set $A$, we define also $C_{D}(x, A)=\min \left\{C_{D}(x, y): y \in A\right\}$.

REMARK. The first formula for the renormalized communication cost (corresponding to the case $x \in D, y \notin D$ ) is the formula given by Wentzell and Freidlin.

REMARK. If $D$ is empty we have $\tau(\emptyset, 1)=1$ and $C_{\emptyset}(x, y)=V(x, y)$ for any $x, y$ in $E$.

Lemma 4.3. For $x \notin D$ and $y \notin D$,

$$
C_{D}(x, y)=\min \left\{V(x, z)+C_{D}(z, y): z \in D \cup\{y\}\right\} .
$$

For $x \in D, y \in D$ and $z \notin D$,

$$
C_{D}(x, z) \leq C_{D}(x, y)+C_{D}(y, z) .
$$


Proof. The first equation is a direct consequence of the expression of the costs $C_{D}(x, y)$ and $C_{D}(z, y)$ in terms of graphs. A probabilistic proof is also possible: let $x, y$ belong to $D^{c}$; we have

$$
\begin{aligned}
& P_{\beta}\left(X_{\tau(D, 1)}=y / X_{0}=x\right)= \\
& \sum_{z \in D \cup\{y\}} P_{\beta}\left(X_{\tau(D)}=y / X_{0}=z\right) P_{\beta}\left(X_{1}=z / X_{0}=x\right)
\end{aligned}
$$

and the first equation follows letting $\beta$ tend to infinity.

Now let $x \in D, y \in D$ and $z \in D^{c}$. We can decompose $P_{\beta}\left(X_{\tau(D)}=z / X_{0}=\right.$ $x$ ) as

$$
\begin{aligned}
P_{\beta}\left(X_{\tau(D \backslash\{y\})}=y,\right. & \left.X_{\tau(D)}=z / X_{0}=x\right)+P_{\beta}\left(X_{\tau(D \backslash\{y\})}=z / X_{0}=x\right) \\
& \geq P_{\beta}\left(X_{\tau(D \backslash\{y\})}=y, X_{\tau(D)}=z / X_{0}=x\right) \\
& =P_{\beta}\left(X_{\tau(D \backslash\{y\})}=y / X_{0}=x\right) P_{\beta}\left(X_{\tau(D)}=z / X_{0}=y\right) .
\end{aligned}
$$

Letting $\beta$ tend to infinity we obtain

$$
C_{D}(x, z) \leq C_{D \backslash\{y\}}(x, y)+C_{D}(y, z) .
$$

Moreover by convention $C_{D \backslash\{y\}}(x, y)=C_{D}(x, y)$.

For another construction of the renormalized communication cost, see Trouvé (1993, 1996a, 1996b).

Proposition 4.4. (properties of the renormalized communication cost) For any subset $D$ of $E$ and for any point $x$ in $E$, the cost $C_{D}\left(x, D^{c}\right)$ is null. For any $x, y$, the set function $A \mapsto C_{A}(x, y)$ is non increasing i.e.

$$
A \subset B \quad \Longrightarrow \quad C_{A}(x, y) \geq C_{B}(x, y) .
$$

Proof. These properties are easy consequences of the probabilistic definition of the renormalized cost. Let us prove the last assertion. First we have $C_{A}(x, y)=C_{A \backslash\{y\}}(x, y), C_{B}(x, y)=C_{B \backslash\{y\}}(x, y)$ and we may thus assume that $y$ is in $B^{c}$. We have the inclusion $B^{c} \subset A^{c}$ so that for $i=0$ or $i=1$,

$$
\left\{X_{\tau(A, i)}=y\right\} \subset\left\{X_{\tau(B, i)}=y\right\} \Longrightarrow P_{\beta}\left(X_{\tau(A, i)}=y\right) \leq P_{\beta}\left(X_{\tau(B, i)}=y\right)
$$

and finally $C_{A}(x, y) \geq C_{B}(x, y)$.

Definition 4.5. (virtual energy) Lemma 3.6 implies that for any $x$ in $E$,

$$
\begin{aligned}
\lim _{\beta \rightarrow \infty}-\frac{1}{\beta} & \ln \mu_{\beta}(x)=W(x) \\
& =\min \{V(g): g \in G(\{x\})\}-\min \{V(g): g \in G(\{y\}), y \in E\} .
\end{aligned}
$$

The quantity $W(x)$ is called the virtual energy of $x$.

For an arbitrary set $D$, we define its virtual energy $W(D)$ by

$$
W(D)=\min \{W(x): x \in D\} .
$$


The bottom $F(D)$ of $D$ is the set of points of $D$ with virtual energy $W(D)$ i.e.

$$
F(D)=\{x \in D: W(x)=W(D)\} .
$$

Definition 4.6. (localized virtual energy) For any subset $D$ of $E$, any point $x$ of $E$ and any $y$ in $D$, lemma 3.5 implies the existence of the limit

$$
\lim _{\beta \rightarrow \infty}-\frac{1}{\beta} \ln \sum_{n=0}^{\infty} P_{\beta}\left(X_{n}=y, \tau(D, 1)>n / X_{0}=x\right)=W_{D}(x, y) .
$$

The quantity $W_{D}(x, y)$ is the logarithmic rate of the potential of the Markov chain starting from $x$ and killed outside $D$. (Note that we do not use $W_{D}(x, y)$ with the same meaning as Freidlin and Wentzell (1984).)

The expression of $W_{D}(x, y)$ in terms of graphs is for any $x$ in $D$ and $y$ in $D$,

$$
W_{D}(x, y)=\min \left\{V(g): g \in G_{x, y}\left(D^{c} \cup\{y\}\right)\right\}-\min \left\{V(g): g \in G\left(D^{c}\right)\right\}
$$

and for any $x$ in $D^{c}$ and $y$ in $D$,

$$
\begin{aligned}
W_{D}(x, y)= & \min \left\{V(x, z)+V(g): z \in D, g \in G_{z, y}\left(D^{c} \cup\{y\}\right)\right\} \\
& \quad-\min \left\{V(g): g \in G\left(D^{c}\right)\right\} \\
= & \min \left\{V(x, z)+W_{D}(z, y): z \in D\right\} .
\end{aligned}
$$

LEMMA 4.7. (link between the localized virtual energy and the renormalized cost) Let $D$ be an arbitrary subset of $E, x$ in $E$ and $y$ in $D$. Then

$$
W_{D}(x, y)+C_{D \backslash\{y\}}\left(y, D^{c}\right)=C_{D \backslash\{y\}}(x, y) .
$$

Proof. Let us assume first that $x$ is in $D$. We have

$$
W_{D}(x, y)=\min \left\{V(g): g \in G_{x, y}\left(D^{c} \cup\{y\}\right)\right\}-\min \left\{V(g): g \in G\left(D^{c}\right)\right\}
$$

and

$C_{D \backslash\{y\}}\left(y, D^{c}\right)=\min \left\{V(g): g \in G\left(D^{c}\right)\right\}-\min \left\{V(g): g \in G\left(D^{c} \cup\{y\}\right)\right\}$.

Thus

$$
\begin{aligned}
W_{D}(x, y)+C_{D \backslash\{y\}}\left(y, D^{c}\right) & =\min \left\{V(g): g \in G_{x, y}\left(D^{c} \cup\{y\}\right)\right\} \\
& \quad-\min \left\{V(g): g \in G\left(D^{c} \cup\{y\}\right)\right\} \\
& =C_{D \backslash\{y\}}(x, y) .
\end{aligned}
$$

When $x$ is in $D^{c}$ we can write

$$
\begin{aligned}
W_{D}(x, y)+C_{D \backslash\{y\}}\left(y, D^{c}\right) & =\min \left\{V(x, z)+W_{D}(z, y): z \in D\right\} \\
& +C_{D \backslash\{y\}}\left(y, D^{c}\right) \\
& =\min \left\{V(x, z)+C_{D \backslash\{y\}}(z, y): z \in D\right\} \\
= & C_{D \backslash\{y\}}(x, y)
\end{aligned}
$$


which is the desired identity.

REMARK. A probabilistic proof is also possible, starting from the identity

$$
\begin{array}{r}
\left(\sum_{n=0}^{\infty} P_{\beta}\left(X_{n}=y, \tau(D, 1)>n / X_{0}=x\right)\right) P_{\beta}\left(X_{\tau(D \backslash\{y\}, 1)} \notin D / X_{0}=y\right)= \\
P_{\beta}\left(X_{\tau(D \backslash\{y\})}=y / X_{0}=x\right) .
\end{array}
$$

In other terms, the probability to escape from $D$ after having visited $y$ is equal to the probability to visit $y$ before leaving $D$ !

DEFInItion 4.8. (height of a set) The height $H(D)$ of the set $D$ is defined by

$$
H(D)=\max _{x \in D} \lim _{\beta \rightarrow \infty} \frac{1}{\beta} \ln E_{\beta}\left(\tau(D) / X_{0}=x\right) .
$$

By lemma 3.8 this limit exists and is equal to

$$
H(D)=-\min \left\{V(g): x \in D, g \in G\left(x \not \rightarrow D^{c}\right)\right\}+\min \left\{V(g): g \in G\left(D^{c}\right)\right\} .
$$

Definition 4.9. (boundary) For a subset $D$ of $E$ we define its bound$\operatorname{ary} B(D)$

$$
B(D)=\{y: y \notin D, \exists x \in D \quad V(x, y)<\infty\}
$$

and its principal boundary $\widetilde{B}(D)$

$$
\widetilde{B}(D)=\left\{y \in B(D): \exists x \in D \quad \lim _{\beta \rightarrow \infty}-\frac{1}{\beta} \ln P_{\beta}\left(X_{\tau(D)}=y / X_{0}=x\right)=0\right\} .
$$

REMARK. The principal boundary of a set $D$ may equivalently be defined by

$$
\widetilde{B}(D)=\left\{y \in B(D): \exists x \in D \quad C_{D}(x, y)=0\right\} .
$$

Definition 4.10. (cycle) A cycle $\pi$ is a subset of $E$ which is either reduced to a point or satisfies

$$
\forall x, y \in \pi, \quad x \neq y, \quad \lim _{\beta \rightarrow \infty}-\frac{1}{\beta} \ln P_{\beta}\left(X_{\tau(\pi \backslash\{y\})} \neq y / X_{0}=x\right)>0 .
$$

The set of cycles is a tree for the set inclusion, two cycles being either disjoint or comparable for the inclusion relation. As said in the introduction, the chain goes to and fro an exponential number of times between any pair of states in a cycle before leaving it. This is a simple consequence of the probabilistic definition of the cycles we chose to use here, and explains why the exit time and point from a cycle is weakly dependent at low temperature from the initial conditions. This is what states the next proposition.

Proposition 4.11. (mixing properties of cycles) Let $\pi$ be a cycle. For any points $x, y$ in $\pi$, the cost $C_{\pi}(x, y)$ is null.

For any subset $D$ of $E$ containing $\pi$ and for any $x, y$ in $\pi$, we have for any $z$ in $E$

$$
\begin{gathered}
W_{D}(z, x)-W_{D}(z, y)=W(x)-W(y) \\
C_{D}(x, z)=C_{D}(y, z), \quad C_{D}(z, x)=C_{D}(z, y)
\end{gathered}
$$

and for any $z$ in $D$, we have $W_{D}(x, z)=W_{D}(y, z)$. 
As a consequence, we can define without ambiguity the renormalized costs $C_{D}(\pi, z), C_{D}(z, \pi)$ for any cycle $\pi$ included in $D$ and any point $z$ in $E$, as well as $C_{D}\left(\pi_{1}, \pi_{2}\right)$ for any cycles $\pi_{1}, \pi_{2}$ included in $D$.

Definition 4.12. (maximal partition) Let $D$ be a subset of $E$. The partition of $D$ into its maximal subcycles is denoted by $\mathcal{M}(D)$. For $x$ in $D$ we denote by $\pi(x, D)$ the unique cycle of $\mathcal{M}(D)$ containing $x$ (for $x$ in $D^{c}$ we make the convention that $\pi(x, D)=\{x\})$.

Remark. In case $D$ is a cycle, we have $\mathcal{M}(D)=\{D\}$.

The relevance of the cycles of $\mathcal{M}(D)$ for the study of the behaviour of the Markov chain in $D$ will already appear in the next lemma. It can also be understood from the remark that the distribution of the exit time and point from $D$ knowing that the chain started from state $x$ will only depend at low temperature on the component of the maximal partition of $D$ to which $x$ belongs.

Notation. Let $g$ be a graph and $A$ a subset of $E$, we denote by $\left.g\right|_{A}$ the restriction of $g$ to $A$, obtained by deleting from $g$ all the arrows starting outside from $A$.

Lemma 4.13. Let $D$ be an arbitrary subset of $E$. We have

$$
\min \left\{V(g): g \in G\left(D^{c}\right)\right\}=\sum_{\pi \in \mathcal{M}(D)} \min \left\{V(g): g \in G\left(\pi^{c}\right)\right\}
$$

Moreover for any graph $\widehat{g}$ in $G\left(D^{c}\right)$ realizing the minimum $\min \{V(g): g \in$ $\left.G\left(D^{c}\right)\right\}$ and any cycle $\pi$ of $\mathcal{M}(D)$, we have $V\left(\left.\widehat{g}\right|_{\pi}\right)=\min \{V(g): g \in$ $\left.G\left(\pi^{c}\right)\right\}$ and $\left.\widehat{g}\right|_{\pi}$ has only one arrow with endpoint outside of $\pi$.

Proof. Let us prove first that any $\widehat{g}$ realizing the $\operatorname{minimum} \min \{V(g): g \in$ $\left.G\left(\pi^{c}\right)\right\}$ has only one arrow with endpoint outside of $\pi$. For this purpose, let us assume that $(x \rightarrow y) \in \widehat{g},\left(x^{\prime} \rightarrow y^{\prime}\right) \in \widehat{g}$ with $x, x^{\prime} \in \pi, y, y^{\prime} \in \pi^{c}$ and $x \neq x^{\prime}$. The graph $\widehat{g} \backslash\left\{\left(x^{\prime} \rightarrow y^{\prime}\right)\right\}$ would belong to $G_{x, y}\left(\pi^{c} \cup\left\{x^{\prime}\right\}\right)$ and would satisfy

$$
V\left(\widehat{g} \backslash\left\{\left(x^{\prime} \rightarrow y^{\prime}\right)\right\}\right)=\min \left\{V(g): g \in G\left(\pi^{c} \cup\left\{x^{\prime}\right\}\right)\right\},
$$

therefore we would have that $y$ belongs to $\widetilde{B}\left(\pi \backslash\left\{x^{\prime}\right\}\right) \cap \pi^{c}$ which is in contradiction with the fact that $\pi$ is a cycle.

Now let $\widehat{g}$ be any graph of $G\left(D^{c}\right)$. For any $\pi$ in $\mathcal{M}(D)$ let $\left.\widehat{g}\right|_{\pi}$ denote its restriction to $\pi$ (obtained by removing arrows with starting point outside $\pi$ ). Clearly the graph $\left.\widehat{g}\right|_{\pi}$ belongs to $G\left(\pi^{c}\right)$. Therefore

$$
V(\widehat{g})=\sum_{\pi \in \mathcal{M}(D)} V\left(\left.\widehat{g}\right|_{\pi}\right) \geq \sum_{\pi \in \mathcal{M}(D)} \min \left\{V(g): g \in G\left(\pi^{c}\right)\right\}
$$

and there is equality if and only if for each $\pi$ in $\mathcal{M}(D)$ we have $V\left(\left.\widehat{g}\right|_{\pi}\right)=$ $\min \left\{V(g): g \in G\left(\pi^{c}\right)\right\}$. Thus to end the proof of the lemma, all we have to do is to build a graph $\widehat{g}$ in $G\left(D^{c}\right)$ such that for any $\pi$ in $\mathcal{M}(D)$, $V\left(\left.\widehat{g}\right|_{\pi}\right)=\min \left\{V(g): g \in G\left(\pi^{c}\right)\right\}$. For this purpose, let us consider the 
graph $\mathcal{G}$ over the set $\mathcal{M}(D) \cup D^{c}$ (the points of $D^{c}$ being identified with one point cycles) defined by

$$
\left(\pi_{1} \rightarrow \pi_{2}\right) \in \mathcal{G} \Longleftrightarrow \widetilde{B}\left(\pi_{1}\right) \cap \pi_{2} \neq \emptyset \quad \Longleftrightarrow \quad C_{\pi_{1}}\left(\pi_{1}, \pi_{2}\right)=0 .
$$

Let $\left.\mathcal{G}\right|_{\mathcal{M}(D)}$ be its restriction to the cycles of $\mathcal{M}(D)$ (that is we only keep the arrows of $\mathcal{G}$ whose starting point is a cycle of $\mathcal{M}(D))$. This restriction has no stable irreducible component in $\mathcal{M}(D)$. Indeed such a component could not be reduced to one cycle, because no cycle (and in fact no set) has an empty principal boundary, and on the other hand, if it were made of more than one cycle, the union of these cycles would be a cycle of $D$, and this would contradict the maximality of the elements of $\mathcal{M}(D)$. Consequently, we can extract from $\left.\mathcal{G}\right|_{\mathcal{M}(D)}$ a spanning collection of oriented trees $\mathcal{H}$ belonging to $G\left(\mathcal{M}(D)^{c}\right)$, that is a graph on $\mathcal{M}(D) \cup D^{c}$ such that

- each cycle of $\mathcal{M}(D)$ is the starting point of exactly one arrow;

- there is no arrow starting from $D^{c}$;

- there is no loop in $\mathcal{H}$.

Now for each $\pi$ in $\mathcal{M}(D)$, considering $\pi^{\prime}$ such that $\left(\pi \rightarrow \pi^{\prime}\right)$ is in $\mathcal{H}$, we can choose $y$ in $\widetilde{B}(\pi) \cap \pi^{\prime}$ (because $\mathcal{H} \subset \mathcal{G}$ ) and find a graph $\widehat{g}_{\pi}$ in $G_{x, y}\left(\pi^{c}\right)$, where $x$ is some point of $\pi$, such that $V\left(\widehat{g}_{\pi}\right)=\min \left\{V(g): g \in G\left(\pi^{c}\right)\right\}$ (because $y$ is in $\widetilde{B}(\pi)$ ). Then we know that $y$ is the only endpoint of the arrows of $\widehat{g}_{\pi}$ lying outside of $\pi$. Let $\widehat{g}$ be the union of all the graphs $\widehat{g}_{\pi}, \pi \in$ $\mathcal{M}(D)$. The graph $\widehat{g}$ has no loop, because any loop in $\widehat{g}$ would correspond to a loop in $\mathcal{H}$. Therefore $\widehat{g}$ belongs to $G\left(D^{c}\right)$ and answers the question.

The formula of lemma 4.13 is very useful to perform several computations of renormalized costs and heights of sets. As an application, we compute the cost appearing in lemma 4.7 above.

Proposition 4.14. For any subset $D$ of $E$ and $s$ in $D$ we have

$$
C_{D \backslash\{s\}}\left(s, D^{c}\right)=H(\pi(s, D))+W(\pi(s, D))-W(s)
$$

(where $\pi(s, D)$ is the greatest cycle included in $D$ and containing s.)

Proof. As we already noticed in the proof of lemma 4.7 , we have

$C_{D \backslash\{s\}}\left(s, D^{c}\right)=\min \left\{V(g): g \in G\left(D^{c}\right)\right\}-\min \left\{V(g): g \in G\left(D^{c} \cup\{s\}\right)\right\}$.

Using lemma 4.13 we obtain that the cost $C_{D \backslash\{s\}}\left(s, D^{c}\right)$ is equal to

$$
\begin{aligned}
& \sum_{\pi \in \mathcal{M}(D)} \min \left\{V(g): g \in G\left(\pi^{c}\right)\right\}-\sum_{\pi \in \mathcal{M}(D \backslash\{s\})} \min \left\{V(g): g \in G\left(\pi^{c}\right)\right\} \\
= & \min \left\{V(g): g \in G\left(\pi(s, D)^{c}\right)\right\}-\sum_{\pi \in \mathcal{M}(\pi(s, D) \backslash\{s\})} \min \left\{V(g): g \in G\left(\pi^{c}\right)\right\} \\
= & \min \left\{V(g): g \in G\left(\pi(s, D)^{c}\right)\right\}-\min \left\{V(g): g \in G\left(\pi(s, D)^{c} \cup\{s\}\right)\right\} .
\end{aligned}
$$

Let $e$ belong to $F(\pi(s, D))$. We have also that the quantity (from the first equation of proposition 4.11)

$$
\min \left\{V(g): g \in G\left(\pi(s, D)^{c} \cup\{s\}\right)\right\}-\min \left\{V(g): g \in G\left(\pi(s, D)^{c} \cup\{e\}\right)\right\}
$$


is equal to $W(s)-W(e)$ (i.e. the computation of a difference of virtual energies can be done within a cycle containing the points) so that finally

$$
\begin{gathered}
C_{D \backslash\{s\}}\left(s, D^{c}\right)=\min \left\{V(g): g \in G\left(\pi(s, D)^{c}\right)\right\} \\
-\min \left\{V(g): g \in G\left(\pi(s, D)^{c} \cup\{e\}\right)\right\}+W(\pi(s, D))-W(s) \\
=H(\pi(s, D))+W(\pi(s, D))-W(s) .
\end{gathered}
$$

Corollary 4.15. For any subset $D$ of $E$ and for any $s$ in $D$, we have

$$
C_{D \backslash\{s\}}\left(s, D^{c}\right)=C_{\pi(s, D) \backslash\{s\}}\left(s, \pi(s, D)^{c}\right) .
$$

Proof. We apply proposition 4.14 to the set $D=\pi(s, D)$. Since the cycle $\pi(s, \pi(s, D)$ ) coincides with $\pi(s, D)$ (see the remark after definition 4.12 ), we see that the $\operatorname{cost} C_{\pi(s, D) \backslash\{s\}}\left(s, \pi(s, D)^{c}\right)$ is also equal to $H(\pi(s, D))+$ $W(\pi(s, D))-W(s)$.

\section{THE EXIT CYCLE PATH}

We will study how the Markov chain $\left(X_{n}\right)_{n \in \mathbb{N}}$ jumps between the cycles of $\mathcal{M}(D \backslash G)$, the partition of $D \backslash G$ into its maximal subcycles, after its last visit to $G$.

DEFINITION 5.1. (cycle path) We define recursively a sequence of random times and cycles:

$$
\begin{array}{ll}
\tau_{-1}=\theta(G, \tau(D)), & \pi_{-1}=\left\{X_{\tau_{-1}}\right\}, \\
\tau_{0}=\tau_{-1}+1, & \pi_{0}=\pi\left(X_{\tau_{0}}, D \backslash G\right), \\
\tau_{1}=\tau\left(\pi_{0}, \tau_{0}\right), & \pi_{1}=\pi\left(X_{\tau_{1}}, D \backslash G\right), \\
\quad \vdots & \vdots \\
\tau_{k}=\tau\left(\pi_{k-1}, \tau_{k-1}\right), & \pi_{k}=\pi\left(X_{\tau_{k}}, D \backslash G\right), \\
\quad \vdots & \vdots \\
\tau_{r}=\tau(D), & \pi_{r}=\left\{X_{\tau_{r}}\right\} .
\end{array}
$$

Notice that the length $r$ is itself random, since $r$ is defined by the equality $\tau_{r}=\tau(D)$.

The sequence $\left(\pi_{-1}, \pi_{0}, \ldots, \pi_{r-1}, \pi_{r}\right)$ is called the cycle path of $\left(X_{n}\right)$ relative to $D, G$ and denoted by $\pi(X, D, G)$. It is a random variable with values in the cycle path space

$$
\begin{aligned}
& \Psi(D, G)=\{\{y\}: y \in G\} \times \\
& \bigcup_{r=0}^{\infty}\left\{\left(\pi_{0}, \ldots, \pi_{r-1}\right) \in \mathcal{M}(D \backslash G)^{r}: \pi_{k} \neq \pi_{k-1}, 1 \leq k<r\right\} \times\left\{\{z\}: z \in D^{c}\right\}
\end{aligned}
$$

of finite sequences of cycles starting in $G$, traveling through $\mathcal{M}(D \backslash G)$ and ending in $D^{c}$. We define a cost function $V_{x}(D, G)$ on the space $\Psi(D, G)$ by

$$
\begin{aligned}
V_{x}(D, G)\left(\{y\}, \pi_{0}, \ldots, \pi_{r-1},\{z\}\right) & = \\
& W_{D}(x, y)+V\left(y, \pi_{0}\right)+\sum_{k=1}^{r-1} C_{\pi_{k-1}}\left(\pi_{k-1}, \pi_{k}\right)+C_{\pi_{r-1}}\left(\pi_{r-1}, z\right)
\end{aligned}
$$


where we recall that by definition,

$$
V\left(y, \pi_{0}\right)=\min _{u \in \pi_{0}} V(y, u), \quad C_{\pi_{k-1}}\left(\pi_{k-1}, \pi_{k}\right)=\min _{v \in \pi_{k}} C_{\pi_{k-1}}\left(\pi_{k-1}, v\right) .
$$

Since the sets $D$ and $G$ will be fixed in the sequel, we will drop them in the notation whenever no confusion is possible: for instance we will write $V_{x}, \Psi, \pi(X)$ instead of $V_{x}(D, G), \Psi(D, G), \pi(X, D, G)$.

Theorem 5.2. (estimation of the probability of a cycle path) There exists a positive constant $K_{1}$ (depending only on the cardinality of $D \backslash G$ ) such that for any exit cycle path $\left(\{y\}, \pi_{0}, \ldots, \pi_{r-1},\{z\}\right)$ in $\Psi(D, G)$ and any $\beta$ we have

$$
\begin{aligned}
& \left(K_{1}^{-1} a(\beta)^{K_{1}}\right)^{r+1} \exp -\beta V_{x}\left(\{y\}, \pi_{0}, \ldots, \pi_{r-1},\{z\}\right) \\
& \leq P_{\beta}\left(\pi(D, G)=\left(\{y\}, \pi_{0}, \ldots, \pi_{r-1},\{z\}\right) / X_{0}=x\right) \leq \\
& \left(K_{1} a(\beta)^{-K_{1}}\right)^{r+1} \exp -\beta V_{x}\left(\{y\}, \pi_{0}, \ldots, \pi_{r-1},\{z\}\right) .
\end{aligned}
$$

Proof. Conditioning by the last visit of the chain to the set $G$ and applying the Markov property we get

$$
\begin{aligned}
& P_{\beta}\left(\pi(D, G)=\left(\{y\}, \pi_{0}, \ldots, \pi_{r-1},\{z\}\right) / X_{0}=x\right)= \\
& \sum_{n=0}^{\infty} P_{\beta}\left(X_{n}=y, \tau(D)>n / X_{0}=x\right) \prod_{k=0}^{r} P_{\beta}\left(X_{\tau_{k}} \in \pi_{k} / X_{\tau_{l}} \in \pi_{l},-1 \leq l<k\right) .
\end{aligned}
$$

By lemma 3.5, there exist positive constants $C_{1}, C_{1}^{\prime}$ such that

$$
\begin{aligned}
C_{1}^{\prime} a(\beta)^{C_{1}} \exp -\beta W_{D}(x, y) \leq \sum_{n=0}^{\infty} P_{\beta}\left(X_{n}=\right. & \left.y, \tau(D)>n / X_{0}=x\right) \\
& \leq C_{1}^{\prime-1} a(\beta)^{-C_{1}} \exp -\beta W_{D}(x, y) .
\end{aligned}
$$

Moreover the first term of the product in formula (5.1) satisfies

$$
\begin{aligned}
a(\beta) \exp -\beta V\left(y, \pi_{0}\right) \leq P_{\beta}\left(X_{\tau_{0}} \in \pi_{0} / X_{\tau_{-1}}\right. & =y) \\
& \leq a(\beta)^{-1}\left|\pi_{0}\right| \exp -\beta V\left(y, \pi_{0}\right)
\end{aligned}
$$

and for $k \geq 1$ we have

$$
\begin{aligned}
& P_{\beta}\left(X_{\tau_{k}} \in \pi_{k} / X_{\tau_{l}} \in \pi_{l},-1 \leq l<k\right)= \\
& \sum_{u \in \pi_{k-1}} P_{\beta}\left(X_{\tau_{k}} \in \pi_{k} / X_{\tau_{k-1}}=u\right) P_{\beta}\left(X_{\tau_{k-1}}=u / X_{\tau_{l}} \in \pi_{l},-1 \leq l<k\right) .
\end{aligned}
$$

Yet there exists a positive constant $C_{2}$ such that for any $u$ in $\pi_{k-1}$,

$$
\begin{aligned}
& C_{2}^{-1} a(\beta)^{C_{2}} \exp -\beta C_{\pi_{k-1}}\left(\pi_{k-1}, \pi_{k}\right) \\
& \leq P_{\beta}\left(X_{\tau_{k}} \in \pi_{k} / X_{\tau_{k-1}}=u\right) \leq \\
& C_{2} a(\beta)^{-C_{2}} \exp -\beta C_{\pi_{k-1}}\left(\pi_{k-1}, \pi_{k}\right)
\end{aligned}
$$


whence, substituting this inequality in the previous equation and summing over $u \in \pi_{k-1}$, we get

$$
\begin{aligned}
C_{2}^{-1} a(\beta)^{C_{2}} \exp -\beta C_{\pi_{k-1}}\left(\pi_{k-1}, \pi_{k}\right) & \\
\leq & P_{\beta}\left(X_{\tau_{k}} \in \pi_{k} / X_{\tau_{l}} \in\right. \\
& \left.\pi_{l},-1 \leq l<k\right) \leq \\
& C_{2} a(\beta)^{-C_{2}} \exp -\beta C_{\pi_{k-1}}\left(\pi_{k-1}, \pi_{k}\right) .
\end{aligned}
$$

and formula (5.1) yields the result.

The cost of a cycle path includes the sum of the costs of each of its arrows $\left(\pi_{k-1} \rightarrow \pi_{k}\right)$. Thus any path of bounded cost has a bounded number of arrows of positive cost. However it may have an arbitrary large number of arrows of null cost if there exist loops of null cost.

Definition 5.3. The cycle path $\left(\{y\}, \pi_{0}, \ldots, \pi_{r-1},\{z\}\right)$ is optimal knowing that $X_{0}=x$ if its $\operatorname{cost} V_{x}\left(\{y\}, \pi_{0}, \ldots, \pi_{r-1},\{z\}\right)$ is equal to the infimum

$$
\inf \left\{V_{x}\left(\psi_{-1}, \ldots, \psi_{s-1},\{z\}\right): s \in \mathbb{N},\left(\psi_{-1}, \ldots, \psi_{s-1},\{z\}\right) \in \Psi(D, G)\right\} .
$$

Let $\mathcal{G}_{x}$ be the graph containing the arrows of all optimal paths knowing that $X_{0}=x$.

Proposition 5.4. The cycle path $\left(\pi_{-1}, \ldots, \pi_{r}\right)$ is optimal knowing that $X_{0}=x$ if and only if it is contained in the optimal graph $\mathcal{G}_{x}$ (that is, all its arrows do appear in the optimal graph).

Remark. That any optimal path belongs to $\mathcal{G}_{x}$ is obvious. The interesting point is that each path contained in the optimal graph $\mathcal{G}_{x}$ yields also an optimal path knowing $X_{0}=x$.

Proof. Let us define the cost of a beginning path $\left(\pi_{-1}, \pi_{0}, \ldots, \pi_{k}\right)$ where the $\pi_{k}$ 's belong to $\mathcal{M}(D \backslash G)$ by

$$
V_{x}\left(\pi_{-1}, \pi_{0}, \ldots, \pi_{k}\right)=W_{D}\left(x, \pi_{-1}\right)+V\left(\pi_{-1}, \pi_{0}\right)+\sum_{l=1}^{k} C_{\pi_{l-1}}\left(\pi_{l-1}, \pi_{l}\right) .
$$

Then for any optimal path $\left(\pi_{-1}, \ldots, \pi_{r}\right)$ and any $k<r,\left(\pi_{-1}, \ldots, \pi_{k}\right)$ is optimal among the paths ending in $\pi_{k}$. Conversely, assume that $\left(\pi_{-1}, \ldots, \pi_{r}\right)$ belongs to $\mathcal{G}_{x}$ i.e. that all the arrows of the path do appear in the graph $\mathcal{G}_{x}$. We prove by induction that $\left(\pi_{-1}, \ldots, \pi_{k}\right)$ is optimal among the paths ending in $\pi_{k}$. Suppose the result is true at rank $k-1$. By the very definition of the optimal graph $\mathcal{G}_{x}$, there exists an optimal path $\gamma$ such that the arrow $\left(\pi_{k-1}, \pi_{k}\right)$ is in $\gamma$. Let $\gamma_{k-1}$ be the path $\gamma$ truncated at $\pi_{k-1}$ and $\gamma_{k}$ be the path $\gamma$ truncated at $\pi_{k}$. By the induction hypothesis $V_{x}\left(\gamma_{k-1}\right)=$ $V_{x}\left(\pi_{-1}, \ldots, \pi_{k-1}\right)$ so that $V_{x}\left(\gamma_{k}\right)=V_{x}\left(\pi_{-1}, \ldots, \pi_{k}\right)$. Since $\gamma_{k}$ is optimal, $\left(\pi_{-1}, \ldots, \pi_{k}\right)$ is also optimal.

REMARK. The same kind of proof appears in the study of the well known dynamic programming algorithm.

We study now the link between the cycle path cost and the renormalized communication cost. 
Theorem 5.5. Let $D$ be a domain, let $x$ belong to $D$ and let $y$ be a point of $E$. Let $\pi_{x}$ and $\pi_{y}$ be the cycles of $\mathcal{M}(D)$ containing $x$ and $y$ respectively (if $y$ is not in $D$, we put $\left.\pi_{y}=\{y\}\right)$. The cost $C_{D}(x, y)$ is equal to the infimum

$$
\begin{aligned}
\inf \left\{\sum_{k=1}^{r} C_{\pi_{k-1}}\left(\pi_{k-1}, \pi_{k}\right):\right. & \\
& \left.\quad r \in \mathbb{N}, \pi_{0}=\pi_{x},\left(\pi_{1}, \ldots, \pi_{r-1}\right) \in \mathcal{M}(D)^{r-1}, \pi_{r}=\pi_{y}\right\} .
\end{aligned}
$$

Remark. Proposition 4.11 shows that $C_{D}(x, y)=C_{D}\left(\pi_{x}, \pi_{y}\right)$ is independent of the pair $(x, y)$ chosen in $\pi_{x} \times \pi_{y}$.

REMARK. If we had not used maximal cycles in theorem 5.5 as well as in the construction of the exit cycle path, the renormalized communication cost would have been strictly inferior to the infimum of the cycle paths costs and all the exit cycle paths of finite length would have had an exponentially vanishing probability to be taken during the last excursion. This shows that the maximal cycles of $\mathcal{M}(D)$ represent the right granularity at which the last excursion should be described.

Proof. In case $\pi_{x}=\pi_{y}$, the cost is zero, as well as the infimum. We assume now that $\pi_{x}$ and $\pi_{y}$ are distinct. By definition of the renormalized cost, we have $C_{D}(x, y)=C_{D \backslash\{y\}}(x, y)$ and since $x$ belongs to $D \backslash\{y\}$, then the cost $C_{D \backslash\{y\}}(x, y)$ is equal to

$$
\min \left\{V(g): g \in G_{x, y}\left(D^{c} \cup\{y\}\right)\right\}-\min \left\{V(g): g \in G\left(D^{c} \cup\{y\}\right)\right\} .
$$

Let $\bar{g}$ be a graph realizing the first minimum i.e. $\bar{g}$ belongs to $G_{x, y}\left(D^{c} \cup\{y\}\right)$ and

$$
V(\bar{g})=\min \left\{V(g): g \in G_{x, y}\left(D^{c} \cup\{y\}\right)\right\} .
$$

There exists a path of arrows in $\bar{g}$ leading from $x$ to $y$. Let $\pi_{0} \rightarrow \cdots \rightarrow \pi_{r}$ be the sequence of the successive cycles of $\mathcal{M}(D)$ visited by this path. Since the path of points starts at $x$ and ends at $y$ we have $\pi_{0}=\pi_{x}$ and $\pi_{r}=\pi_{y}$. In addition we remove the loops in this cycle path, in order to obtain a path of distinct cycles. Now

$$
C_{D}(x, y)=V(\bar{g})-\min \left\{V(g): g \in G\left(D^{c} \cup\{y\}\right)\right\} .
$$

Decomposing $\bar{g}$ on the cycles of $\mathcal{M}(D)$ and applying lemma 4.13 we get

$$
\begin{aligned}
C_{D}(x, y)= & \sum_{\pi \in \mathcal{M}(D)} V\left(\left.\bar{g}\right|_{\pi}\right)-\sum_{\pi \in \mathcal{M}(D \backslash\{y\})} \min \left\{V(g): g \in G\left(\pi^{c}\right)\right\} \\
= & \sum_{\pi \in \mathcal{M}(D), \pi \neq \pi_{y}}\left(V\left(\left.\bar{g}\right|_{\pi}\right)-\min \left\{V(g): g \in G\left(\pi^{c}\right)\right\}\right)+V\left(\left.\bar{g}\right|_{\pi_{y}}\right) \\
& -\sum_{\pi \in \mathcal{M}(D \backslash\{y\}), \pi \subset \pi_{y}} \min \left\{V(g): g \in G\left(\pi^{c}\right)\right\} .
\end{aligned}
$$


However the set of cycles $\left\{\pi: \pi \in \mathcal{M}(D \backslash\{y\}), \pi \subset \pi_{y}\right\}$ is exactly the maximal partition $\mathcal{M}\left(\pi_{y} \backslash\{y\}\right)$ whence by applying once more lemma 4.13, the cost $C_{D}(x, y)$ is equal to

$$
\begin{aligned}
\sum_{\pi \in \mathcal{M}(D), \pi \neq \pi_{y}}\left(V\left(\left.\bar{g}\right|_{\pi}\right)-\min \left\{V(g): g \in G\left(\pi^{c}\right)\right\}\right)+V\left(\left.\bar{g}\right|_{\pi_{y}}\right) \\
\\
-\min \left\{V(g): g \in G\left(\pi_{y}^{c} \cup\{y\}\right)\right\} .
\end{aligned}
$$

Since $\bar{g}$ belongs to $G_{x, y}\left(D^{c} \cup\{y\}\right)$, for any $\pi$ in $\mathcal{M}(D) \backslash \pi_{y}$, its restriction $\left.\bar{g}\right|_{\pi}$ belongs to $G\left(\pi^{c}\right)$. Moreover, $\left.\bar{g}\right|_{\pi_{y}}$ belongs to $G\left(\pi_{y}^{c} \cup\{y\}\right)$. As a consequence,

$$
C_{D}(x, y) \geq \sum_{k=0}^{r-1}\left(V\left(\left.\bar{g}\right|_{\pi_{k}}\right)-\min \left\{V(g): g \in G\left(\pi_{k}^{c}\right)\right\}\right)
$$

(where $\pi_{0}, \ldots, \pi_{r}$ is the sequence of cycles previously described).

By construction of the $\pi_{k}$ 's, there are $x_{k}$ in $\pi_{k}$ and $y_{k}$ in $\pi_{k+1}$ such that $\left.\bar{g}\right|_{\pi_{k}}$ is in $G_{x_{k}, y_{k}}\left(\pi_{k}^{c}\right)$, thus $V\left(\left.\bar{g}\right|_{\pi_{k}}\right)-\min \left\{V(g): g \in G\left(\pi_{k}^{c}\right)\right\} \geq C_{\pi_{k}}\left(x_{k}, y_{k}\right)$. We obtain finally the desired inequality

$$
C_{D}(x, y) \geq \sum_{k=0}^{r-1} C_{\pi_{k}}\left(\pi_{k}, \pi_{k+1}\right)
$$

Conversely, let $\pi_{0} \rightarrow \cdots \rightarrow \pi_{r}$ be a cycle path in $\mathcal{M}(D)$ starting at $\pi_{0}=\pi_{x}$, ending at $\pi_{r}=\pi_{y}$ and realizing the infimum of the theorem. Let us introduce the stopping times

$$
\nu_{-1}=0, \quad \nu_{k}=\tau\left(\pi_{k}, \nu_{k-1}\right) \text { for } 0 \leq k<r, \quad \nu_{r}=\tau\left(\pi_{r} \backslash\{y\}, \nu_{r-1}\right) .
$$

We have, putting $\pi_{r+1}=\{y\}$,

$$
\begin{aligned}
& P_{\beta}\left(X_{\tau(D \backslash\{y\})}=y / X_{0}=x\right) \\
& \geq \prod_{k=0}^{r} P_{\beta}\left(X_{\nu_{k}} \in \pi_{k+1} / X_{\nu_{s}} \in \pi_{s+1}, s<k, X_{0}=x\right) \\
& \geq \prod_{k=0}^{r-1} \inf _{u \in \pi_{k}} P_{\beta}\left(X_{\tau\left(\pi_{k}\right)} \in \pi_{k+1} / X_{0}=u\right) \times \inf _{u \in \pi_{r}} P_{\beta}\left(X_{\tau\left(\pi_{r} \backslash\{y\}\right)}=y / X_{0}=u\right) .
\end{aligned}
$$

Using the definition of the communication cost and letting $\beta$ tend to infinity we get that

$$
C_{D}(x, y) \leq \sum_{k=0}^{r-1} C_{\pi_{k}}\left(\pi_{k}, \pi_{k+1}\right)
$$

We first obtain a slight improvement of the second equation of lemma 4.3. Corollary 5.6. For $x$ in $D$ and $y$ in $D^{c}$, the cost $C_{D}(x, y)$ is equal to

$$
C_{D}(x, y)=\min \left\{C_{D}(x, z)+C_{\pi(z, D)}(z, y): z \in D\right\} .
$$


We apply now theorem 5.5 to the set $D \backslash G$.

Corollary 5.7. For any $y$ in $G$ and $z$ in $D^{c}$, the cost $C_{D \backslash G}(y, z)$ is equal to the infimum

$$
\inf \left\{V\left(y, \pi_{0}\right)+\sum_{k=1}^{r-1} C_{\pi_{k}}\left(\pi_{k}, \pi_{k+1}\right):\left(\{y\}, \pi_{0}, \ldots, \pi_{r-1},\{z\}\right) \in \Psi(D, G)\right\} .
$$

Proof. This is a consequence of the fact that

$$
C_{D \backslash G}(y, z)=\min \left\{V(y, u)+C_{D \backslash G}(u, z): u \in D \backslash G \cup\{z\}\right\}
$$

(lemma 4.3) and of theorem 5.5.

Corollary 5.8. The function $L_{x}$ from $\mathcal{M}(D \backslash G) \cup\left\{\{z\}: z \in D^{c}\right\}$ to $\mathbb{R}^{+}$ defined by

$$
L_{x}(\pi)=\min _{y \in G}\left(W_{D}(x, y)+C_{D \backslash G}(y, \pi)\right)
$$

is non decreasing along the optimal cycle paths knowing that $X_{0}=x$.

\section{THE PRUNED CYCLE PATH}

The estimates in theorem 5.2 are not very satisfactory because their precision depends on the length of the path and also because paths of bounded cost do not necessarily have a bounded length.

In this section we will eliminate the loops in the graph of transitions of null cost between cycles. We define a relation $\rightarrow$ on the cycles of $\mathcal{M}(D \backslash G)$ by

$$
\pi_{1} \rightarrow \pi_{2} \quad \Longleftrightarrow \quad \widetilde{B}\left(\pi_{1}\right) \cap \pi_{2} \neq \emptyset \quad \Longleftrightarrow \quad C_{\pi_{1}}\left(\pi_{1}, \pi_{2}\right)=0 .
$$

The associated equivalence relation $\equiv$ is

$$
\begin{gathered}
\pi \equiv \pi^{\prime} \quad \Longleftrightarrow \quad \exists \pi_{1}, \ldots, \pi_{r}, \pi_{1}^{\prime}, \ldots, \pi_{s}^{\prime} \in \mathcal{M}(D \backslash G) \\
\pi \rightarrow \pi_{1} \rightarrow \cdots \rightarrow \pi_{r} \rightarrow \pi^{\prime} \rightarrow \pi_{1}^{\prime} \rightarrow \cdots \rightarrow \pi_{s}^{\prime} \rightarrow \pi
\end{gathered}
$$

We denote by $\overline{\mathcal{M}}(D \backslash G)$ the partition of $\mathcal{M}(D \backslash G)$ determined by this equivalence relation. Formally, the elements of $\overline{\mathcal{M}}(D \backslash G)$ are sets of cycles, but we rather consider them as subsets of $E$ by identifying $\bar{\pi}$ in $\mathcal{M}(D \backslash G)$ with the set $\{x: \exists \pi \in \bar{\pi}, x \in \pi\}$. For a point $x$ in $D \backslash G$, we denote by $\bar{\pi}(x, D \backslash G)$ the unique element of $\overline{\mathcal{M}}(D \backslash G)$ containing $x$.

For any $\bar{\pi}$ in $\overline{\mathcal{M}}(D \backslash G)$ and for any $y$ in $E$ the cost $C_{\bar{\pi}}(x, y)$ is independent of the point $x$ in $\bar{\pi}$ (by theorem 5.5). We can thus define the $\operatorname{cost} C_{\bar{\pi}}(\bar{\pi}, y)$. Once more, if $A$ is a subset of $E$, we set $C_{\bar{\pi}}(\bar{\pi}, A)=\min \left\{C_{\bar{\pi}}(\bar{\pi}, y): y \in A\right\}$. DEFinition 6.1. (pruned cycle path) We define recursively a sequence of random times and cycles:

$$
\begin{array}{ll}
\bar{\tau}_{-1}=\theta(G, \tau(D)), & \bar{\pi}_{-1}=\left\{X_{\bar{\tau}_{-1}}\right\}, \\
\bar{\tau}_{0}=\bar{\tau}_{-1}+1, & \bar{\pi}_{0}=\bar{\pi}\left(X_{\bar{\tau}_{0}}, D \backslash G\right), \\
\bar{\tau}_{1}=\tau\left(\bar{\pi}_{0}, \bar{\tau}_{0}\right), & \bar{\pi}_{1}=\bar{\pi}\left(X_{\bar{\tau}_{1}}, D \backslash G\right), \\
\quad \vdots & \vdots \\
\bar{\tau}_{k}=\tau\left(\bar{\pi}_{k-1}, \bar{\tau}_{k-1}\right), & \bar{\pi}_{k}=\bar{\pi}\left(X_{\bar{\tau}_{k}}, D \backslash G\right), \\
\quad \vdots & \vdots \\
\bar{\tau}_{r}=\tau(D), & \bar{\pi}_{r}=\left\{X_{\bar{\tau}_{r}}\right\} .
\end{array}
$$


The sequence $\left(\bar{\pi}_{-1}, \bar{\pi}_{0}, \ldots, \bar{\pi}_{r-1}, \bar{\pi}_{r}\right)$ is called the pruned cycle path of $\left(X_{n}\right)$ relative to $D, G$ and is denoted by $\bar{\pi}(X, D, G)$. It is a random variable with values in the pruned cycle path space

$$
\begin{aligned}
& \bar{\Psi}(D, G)=\{\{y\}: y \in G\} \times \\
& \bigcup_{r=0}^{\infty}\left\{\left(\bar{\pi}_{0}, \ldots, \bar{\pi}_{r-1}\right) \in \overline{\mathcal{M}}(D \backslash G)^{r}: \bar{\pi}_{k} \neq \bar{\pi}_{k-1}, 1 \leq k<r\right\} \times\left\{\{z\}: z \in D^{c}\right\}
\end{aligned}
$$

of finite sequences of sets of equivalent cycles starting in $G$, traveling through $\overline{\mathcal{M}}(D \backslash G)$ and ending in $D^{c}$. We define a cost function $\bar{V}_{x}(D, G)$ on the space $\bar{\Psi}(D, G)$ by

$$
\begin{aligned}
\bar{V}_{x}(D, G)\left(\{y\}, \bar{\pi}_{0}, \ldots, \bar{\pi}_{r-1},\{z\}\right) & = \\
W_{D}(x, y)+V\left(y, \bar{\pi}_{0}\right) & +\sum_{k=1}^{r-1} C_{\bar{\pi}_{k-1}}\left(\bar{\pi}_{k-1}, \bar{\pi}_{k}\right)+C_{\bar{\pi}_{r-1}}\left(\bar{\pi}_{r-1}, z\right) .
\end{aligned}
$$

Notice that the pruned cycle path of $\left(X_{n}\right)$ is a partition of the cycle path of $\left(X_{n}\right)$ : it is obtained by regrouping equivalent cycles in $\mathcal{M}(D \backslash G)$. Theorem 5.5 shows that the $V_{x}-$ cost of a cycle path and the $\bar{V}_{x}-\operatorname{cost}$ of the corresponding pruned cycle path are equal i.e. we have $V_{x}(\pi(X, D, G))=$ $\bar{V}_{x}(\bar{\pi}(X, D, G))$.

Theorem 6.2. (estimation of the probability of a pruned cycle path) There exists a positive constant $K_{2}$ (depending only on the cardinality of $D \backslash G$ ) such that for any pruned cycle path $\left(\{y\}, \bar{\pi}_{0}, \ldots, \bar{\pi}_{r-1},\{z\}\right)$ in $\bar{\Psi}(D, G)$ and any $\beta$ we have

$$
\begin{aligned}
& \left(K_{2}^{-1} a(\beta)^{K_{2}}\right)^{r+1} \exp -\beta \bar{V}_{x}\left(\{y\}, \bar{\pi}_{0}, \ldots, \bar{\pi}_{r-1},\{z\}\right) \\
& \leq P_{\beta}\left(\bar{\pi}(D, G)=\left(\{y\}, \bar{\pi}_{0}, \ldots, \bar{\pi}_{r-1},\{z\}\right) / X_{0}=x\right) \leq \\
& \left(K_{2} a(\beta)^{-K_{2}}\right)^{r+1} \exp -\beta \bar{V}_{x}\left(\{y\}, \bar{\pi}_{0}, \ldots, \bar{\pi}_{r-1},\{z\}\right) .
\end{aligned}
$$

Proof. This is the same kind of proof as for theorem 5.2. The only difference is that we now use the estimation (for some constant $C_{4}$ ), for all $u$ in $\bar{\pi}_{k-1}$,

$$
\begin{array}{r}
C_{4}^{-1} a(\beta)^{C_{4}} \exp -\beta C_{\bar{\pi}_{k-1}}\left(\bar{\pi}_{k-1}, \bar{\pi}_{k}\right) \\
\leq P_{\beta}\left(X_{\bar{\tau}_{k}} \in \bar{\pi}_{k} / X_{\bar{\tau}_{k-1}}=u\right) \leq \\
C_{4} a(\beta)^{-C_{4}} \exp -\beta C_{\bar{\pi}_{k-1}}\left(\bar{\pi}_{k-1}, \bar{\pi}_{k}\right) .
\end{array}
$$

The key fact that makes $\bar{V}_{x}$ a good cost function to work with is the following LEMMA 6.3. Let $\left(\bar{\pi}_{0}, \ldots, \bar{\pi}_{r}\right)$ be a sequence such that $\bar{\pi}_{k} \in \overline{\mathcal{M}}(D \backslash G)$, $\bar{\pi}_{k} \neq \bar{\pi}_{k-1}, 1 \leq k \leq r$. There exists a positive constant $\delta$ independent of $\left(\bar{\pi}_{0}, \ldots, \bar{\pi}_{r}\right)$ such that, if $r$ is greater than the cardinality of $\overline{\mathcal{M}}(D \backslash G)$, then

$$
\sum_{k=1}^{r} C_{\bar{\pi}_{k-1}}\left(\bar{\pi}_{k-1}, \bar{\pi}_{k}\right) \geq \delta>0 .
$$


Remark. The graph of the jumps of null cost over $\overline{\mathcal{M}}(D \backslash G)$ has no loop. We could have replaced the cardinality of $\overline{\mathcal{M}}(D \backslash G)$ by the diameter of this graph, that is the number of vertices of its longest paths.

Proof. Since $r$ is greater than or equal to $|\overline{\mathcal{M}}(D \backslash G)|$, two elements $\bar{\pi}_{i}$ and $\bar{\pi}_{j}, 0 \leq i<j \leq r$ of the sequence $\bar{\pi}_{0}, \ldots, \bar{\pi}_{r}$ have to be equal. Since $\bar{\pi}_{i} \neq \bar{\pi}_{i+1}$, then $j>i+1$ and $\bar{\pi}_{i}, \bar{\pi}_{i+1}, \ldots, \bar{\pi}_{j}=\bar{\pi}_{i}$ is a loop. Therefore one of its jumps at least has a positive cost and the sum of the lemma is not smaller than

$$
\delta=\min \left\{C_{\bar{\pi}}(\bar{\pi}, y): \bar{\pi} \in \overline{\mathcal{M}}(D \backslash G), y \in B(\bar{\pi}), C_{\bar{\pi}}(\bar{\pi}, y)>0\right\}
$$

Corollary 6.4. The cost $\bar{V}_{x}$ gives a control on the length of the pruned cycle path i.e.

$$
\forall \lambda \quad \exists R(\lambda) \quad \forall r \geq R(\lambda) \quad \forall\left(\bar{\pi}_{0}, \ldots, \bar{\pi}_{r}\right) \in \bar{\Psi}(D \backslash G) \quad \bar{V}_{x}\left(\bar{\pi}_{0}, \ldots, \bar{\pi}_{r}\right) \geq \lambda .
$$

Corollary 6.5. The cost function $\bar{V}_{x}$ is a good rate function on $\bar{\Psi}$ i.e. its level sets are compact (finite in our situation).

Proof. Corollary 6.4 shows that the sets $\left\{\left(\bar{\pi}_{0}, \ldots, \bar{\pi}_{r}\right): \bar{V}_{x}\left(\bar{\pi}_{0}, \ldots, \bar{\pi}_{r}\right) \leq\right.$ $\lambda$ \} contain only sequences of bounded length and are thus finite.

The length of the cycle path $\pi(X)$ (i.e. the number of jumps it involves) will be denoted by $|\pi(X)|$ in the sequel. For instance $\left|\left(\pi_{0}, \ldots, \pi_{r}\right)\right|=r$.

Proposition 6.6. There exist a positive constant $K_{3}$ and a function $h: \mathbb{N} \rightarrow \mathbb{R}_{+}$such that $\lim _{+\infty} h=+\infty$ and

$$
P_{\beta}\left(|\bar{\pi}(X)| \geq R / X_{0}=x\right) \leq\left(K_{3} a(\beta)^{-K_{3}}\right)^{R} \exp -\beta h(R) .
$$

Proof.

$$
\begin{aligned}
& P_{\beta}\left(|\bar{\pi}(X)| \geq R / X_{0}=x\right) \\
& =\sum_{y, \bar{\pi}_{0}, \ldots, \bar{\pi}_{R}} P_{\beta}\left(\bar{\pi}_{-1}(X)=\{y\}, \bar{\pi}_{0}(X)=\bar{\pi}_{0}, \ldots, \bar{\pi}_{R}(X)=\bar{\pi}_{R} / X_{0}=x\right) \\
& \leq \sum_{y, \bar{\pi}_{0}, \ldots, \bar{\pi}_{R}}\left(K_{2} a(\beta)^{-K_{2}}\right)^{R+1} \times \\
& \quad \operatorname{exp-\beta }\left(W_{D}(x, y)+V\left(y, \bar{\pi}_{0}\right)+\sum_{k=1}^{R} C_{\bar{\pi}_{k-1}}\left(\bar{\pi}_{k-1}, \bar{\pi}_{k}\right)\right) \\
& \leq|G|\left(|\overline{\mathcal{M}}(D \backslash G)| K_{2} a(\beta)^{-K_{2}}\right)^{R+1} \exp -\beta\left([R /|\overline{\mathcal{M}}(D \backslash G)|] \delta+C_{3}\right)
\end{aligned}
$$

where $\delta$ is as in lemma 6.3 and

$$
C_{3}=\inf \left\{W_{D}(x, y)+V\left(y, \bar{\pi}_{0}\right): y \in G, \bar{\pi}_{0} \in \overline{\mathcal{M}}(D \backslash G)\right\} .
$$


Theorem 6.7. (large deviations upper bound) For any positive $\lambda$, there exists a positive constant $K_{4}$ such that for any $\beta$

$$
P_{\beta}\left(V_{x}(\pi(X)) \geq \lambda / X_{0}=x\right) \leq K_{4} a(\beta)^{-K_{4}} \exp -\beta \lambda .
$$

Proof. We decompose the event $\left\{V_{x}(\pi(X)) \geq \lambda\right\}$ according to the length of the associated pruned cycle path. Let $R$ be such that $h(R)>\lambda$. We have

$$
\begin{gathered}
P_{\beta}\left(V_{x}(\pi(X)) \geq \lambda / X_{0}=x\right)=P_{\beta}\left(\bar{V}_{x}(\bar{\pi}(X)) \geq \lambda / X_{0}=x\right) \\
=P_{\beta}\left(\bar{V}_{x}(\bar{\pi}(X)) \geq \lambda,|\bar{\pi}(X)| \geq R / X_{0}=x\right)+ \\
\quad P_{\beta}\left(\bar{V}_{x}(\bar{\pi}(X)) \geq \lambda,|\bar{\pi}(X)|<R / X_{0}=x\right) .
\end{gathered}
$$

The first term in the sum is controlled by proposition 6.6 . The second term is equal to

$$
\sum P_{\beta}\left(\bar{\pi}(X)=\left(\bar{\pi}_{0}, \ldots, \bar{\pi}_{r}\right) / X_{0}=x\right)
$$

where the sum runs over all pruned cycle paths $\left(\bar{\pi}_{0}, \ldots, \bar{\pi}_{r}\right)$ satisfying the conditions $r<R, \bar{V}_{x}\left(\bar{\pi}_{0}, \ldots, \bar{\pi}_{r}\right) \geq \lambda$. For such a path we have by theorem 6.2

$$
P_{\beta}\left(\bar{\pi}(X)=\left(\bar{\pi}_{0}, \ldots, \bar{\pi}_{r}\right) / X_{0}=x\right) \leq K_{2}^{r+1} a(\beta)^{-K_{2}(r+1)} \exp -\beta \lambda
$$

and there is only a finite number of such terms (the length of the paths being bounded). The desired upper bound follows easily.

\section{THE EXIT SADDLE PATH}

In this section, we will study the entrance and exit points of the cycles of the exit cycle path.

DEFINITION 7.1. (saddle path) We define recursively a sequence of random times and points:

$$
\begin{aligned}
& \tau_{0}=\theta(G, \tau(D))+1, \quad s_{0}=X_{\tau_{0}-1}, \quad s_{1}=X_{\tau_{0}}, \\
& \tau_{1}=\tau\left(\pi\left(s_{1}, D \backslash G\right), \tau_{0}\right), \quad s_{2}=X_{\tau_{1}-1}, \quad s_{3}=X_{\tau_{1}}, \\
& \vdots \quad \vdots \quad \vdots \\
& \tau_{k}=\tau\left(\pi\left(s_{2 k-1}, D \backslash G\right), \tau_{k-1}\right), \quad s_{2 k}=X_{\tau_{k}-1}, \quad s_{2 k+1}=X_{\tau_{k}}, \\
& \vdots \\
& \tau_{r}=\tau(D), \\
& \begin{array}{cc}
\vdots & \vdots \\
s_{2 r}=X_{\tau_{r}-1}, & s_{2 r+1}=X_{\tau_{r}} .
\end{array}
\end{aligned}
$$

REMARK. The times $\tau_{i}$ are the same as those used in the definition of the cycle path. We recall also that the length $r$ is random, since $r$ is defined by the equality $\tau_{r}=\tau(D)$.

The sequence $\left(s_{0}, \ldots, s_{2 r+1}\right)$ is called the saddle path of $\left(X_{n}\right)$ relative to $D, G$ and denoted by $\mathcal{S}(X, D, G)$. The saddle path space relative to $D, G$ starting at $x$ is the set

$\mathcal{S}(D, G)=\left\{\left(s_{0}, \ldots, s_{2 r+1}\right): s_{0} \in G, s_{2 r+1} \in D^{c}\right.$, $\forall k \in\{1, \ldots, r\}, s_{2 k-1} \in D \backslash G, s_{2 k} \in \pi\left(s_{2 k-1}, D \backslash G\right), V\left(s_{2 k}, s_{2 k+1}\right)<\infty$,

$$
\left.s_{2 k+1} \in B\left(\pi\left(s_{2 k-1}, D \backslash G\right)\right)\right\} \text {. }
$$


We define a cost function $V_{x}(D, G)$ on the space $\mathcal{S}(D, G)$ by

$$
\begin{aligned}
& V_{x}(D, G)\left(s_{0}, \ldots, s_{2 r+1}\right)=W_{D}\left(x, s_{0}\right)+V\left(s_{0}, s_{1}\right)+ \\
& \sum_{k=1}^{r}\left(W\left(s_{2 k}\right)+V\left(s_{2 k}, s_{2 k+1}\right)-H\left(\pi\left(s_{2 k}, D \backslash G\right)\right)-W\left(\pi\left(s_{2 k}, D \backslash G\right)\right)\right) .
\end{aligned}
$$

Since the sets $D$ and $G$ will be fixed in the sequel, we will drop them in the notation whenever no confusion is possible: for instance we will write $V_{x}$, $\mathcal{S}, \mathcal{S}(X)$ instead of $V_{x}(D, G), \mathcal{S}(D, G), \mathcal{S}(X, D, G)$.

The next lemma estimates the probability of one fixed jump of a saddle path.

Lemma 7.2. (last point and exit point of a cycle) There exists a positive constant $K_{5}$ such that for any cycle $\pi, x, y \in \pi$ and $z \in B(\pi)$,

$$
\begin{aligned}
K_{5}^{-1} a(\beta)^{K_{5}} \exp -\beta(W(y)+V(y, z)-H(\pi)-W(\pi)) \\
\leq \quad P_{\beta}\left(X_{\tau(\pi)}=z, X_{\tau(\pi)-1}=y / X_{0}=x\right) \leq \\
\quad K_{5} a(\beta)^{-K_{5}} \exp -\beta(W(y)+V(y, z)-H(\pi)-W(\pi)) .
\end{aligned}
$$

Proof. By summing over the possible values of the last instant in $\pi$, we obtain

$$
\begin{aligned}
P_{\beta}\left(X_{\tau(\pi)}\right. & \left.=z, X_{\tau(\pi)-1}=y / X_{0}=x\right) \\
& =\sum_{k=0}^{\infty} P_{\beta}\left(\tau(\pi)>k, X_{k}=y, X_{k+1}=z / X_{0}=x\right) \\
& =\left(\sum_{k=0}^{\infty} P_{\beta}\left(\tau(\pi)>k, X_{k}=y / X_{0}=x\right)\right) P_{\beta}\left(X_{1}=z / X_{0}=y\right) .
\end{aligned}
$$

Lemma 3.5 yields the existence of a positive constant $C_{5}$ such that

$$
\begin{aligned}
C_{5}^{-1} a(\beta)^{C_{5}} \exp -\beta W_{\pi}(x, y) \leq \sum_{k=0}^{\infty} P_{\beta}(\tau(\pi)> & \left.k, X_{k}=y / X_{0}=x\right) \\
& \leq C_{5} a(\beta)^{-C_{5}} \exp -\beta W_{\pi}(x, y)
\end{aligned}
$$

By lemma 4.7, we know that $W_{\pi}(x, y)=C_{\pi \backslash\{y\}}(x, y)-C_{\pi \backslash\{y\}}\left(y, \pi^{c}\right)$. Since $\pi$ is a cycle, the cost $C_{\pi \backslash\{y\}}(x, y)$ is null. Moreover proposition 4.14 gives

$$
-W_{\pi}(x, y)=C_{\pi \backslash\{y\}}\left(y, \pi^{c}\right)=H(\pi)+W(\pi)-W(y) .
$$

The required estimation follows easily.

Corolla Ry 7.3. For any cycle $\pi$, any point $x$ in $\pi$ and any point $y$ in $B(\pi)$, we have

$$
C_{\pi}(x, y)=\min \{W(z)+V(z, y): z \in \pi\}-H(\pi)-W(\pi) .
$$


THEorem 7.4. (estimation of the probability of a saddle path) There exists a positive constant $K_{6}$ (depending only on the cardinality of $D \backslash G$ ) such that for any saddle path $\left(s_{0}, \ldots, s_{2 r+1}\right)$ in $\mathcal{S}(D \backslash G)$ and any $\beta$ we have

$$
\begin{aligned}
& \left(K_{6}^{-1} a(\beta)^{K_{6}}\right)^{r+1} \exp -\beta V_{x}\left(s_{0}, \ldots, s_{2 r+1}\right) \\
& \leq P_{\beta}\left(\mathcal{S}(X)=\left(s_{0}, \ldots, s_{2 r+1}\right) / X_{0}=x\right) \leq \\
& \left(K_{6} a(\beta)^{-K_{6}}\right)^{r+1} \exp -\beta V_{x}\left(s_{0}, \ldots, s_{2 r+1}\right) .
\end{aligned}
$$

Proof. If $\mathcal{S}(X)=\left(s_{0}, \ldots, s_{2 r+1}\right)$, the last visit of the chain $\left(X_{n}\right)$ in $G$ occurred at $s_{0}$. Conditioning according to the time of this last visit and then applying the Markov property, we obtain

$$
\begin{aligned}
& P_{\beta}\left(\mathcal{S}(X)=\left(s_{0}, \ldots, s_{2 r+1}\right) / X_{0}=x\right)= \\
& \sum_{k=0}^{\infty} P_{\beta}\left(\mathcal{S}(X)=\left(s_{0}, \ldots, s_{2 r+1}\right), X_{k}=s_{0}, X_{l} \notin G, k<l \leq \tau(D) / X_{0}=x\right)= \\
& \sum_{k=0}^{\infty} P_{\beta}\left(\mathcal{S}(X)=\left(s_{0}, \ldots, s_{2 r+1}\right), X_{k}=s_{0}, X_{l} \notin G, k<l \leq \tau(D) / X_{k}=s_{0}\right) \\
& \times P_{\beta}\left(X_{k}=s_{0}, k<\tau(D) / X_{0}=x\right)= \\
& \left(\sum_{k=0}^{\infty} P_{\beta}\left(X_{k}=s_{0}, k<\tau(D) / X_{0}=x\right)\right) \times \\
& P_{\beta}\left(\mathcal{S}(X)=\left(s_{0}, \ldots, s_{2 r+1}\right), X_{0}=s_{0}, \forall l \quad 0<l \leq \tau(D), X_{l} \notin G / X_{0}=s_{0}\right) .
\end{aligned}
$$

Lemma 3.5 yields the existence of a positive constant $C_{6}$ such that

$$
\begin{aligned}
& C_{6}^{-1} a(\beta)^{C_{6}} \exp -\beta W_{D}\left(x, s_{0}\right) \\
& \leq \sum_{k=0}^{\infty} P_{\beta}\left(X_{k}=s_{0}, k<\tau(D) / X_{0}=x\right) \leq \\
& C_{6} a(\beta)^{-C_{6}} \exp -\beta W_{D}\left(x, s_{0}\right)
\end{aligned}
$$

We now deal with the second term in formula (7.1). By repeated conditioning and applications of the strong Markov property we get

$$
\begin{gathered}
P_{\beta}\left(\mathcal{S}(X)=\left(s_{0}, \ldots, s_{2 r+1}\right), \forall l \quad 0<l \leq \tau(D), X_{l} \notin G / X_{0}=s_{0}\right)= \\
P_{\beta}\left(X_{\tau_{r}}=s_{2 r+1}, X_{\tau_{r}-1}=s_{2 r} / X_{\tau_{r-1}}=s_{2 r-1}\right) \times \cdots \times \\
P_{\beta}\left(X_{\tau_{1}}=s_{3}, X_{\tau_{1}-1}=s_{2} / X_{\tau_{0}}=s_{1}\right) P_{\beta}\left(X_{\tau_{0}}=s_{1}, \tau_{0}=1 / X_{0}=s_{0}\right) \\
=\prod_{k=1}^{r} P_{\beta}\left(X_{\tau}=s_{2 k+1}, X_{\tau-1}=s_{2 k}, \tau=\tau\left(\pi\left(s_{2 k-1}, D \backslash G\right)\right) / X_{0}=s_{2 k-1}\right) \\
\times P_{\beta}\left(X_{1}=s_{1} / X_{0}=s_{0}\right) .
\end{gathered}
$$

Several applications of lemma 7.2 to the above expression, together with formulas (7.1) and (7.2), yield the desired estimation.

We would like to show that the law of the saddle path of $\left(X_{n}\right)$ concentrates on the set of sequences in $\mathcal{S}$ having a null $V_{x}$-cost as $\beta$ goes to infinity. 
Definition 7.5. (optimal saddle) Let $\pi$ be a cycle. A pair $(y, z)$ is an optimal saddle escaping from $\pi$ if

$$
y \in \pi, \quad z \in \widetilde{B}(\pi), \quad W(y)+V(y, z)=W(\pi)+H(\pi) .
$$

Let $\left(s_{0}, \ldots, s_{2 r+1}\right)$ belong to $\mathcal{S}$ and suppose there exists a sequence $\left(t_{0}, \ldots, t_{2 h+1}\right)$ in $D \backslash G$ such that

$$
t_{0} \in \pi\left(s_{1}, D \backslash G\right), \quad t_{1} \in \widetilde{B}\left(\pi\left(s_{1}, D \backslash G\right)\right), \quad t_{2 h+1} \in \pi\left(s_{1}, D \backslash G\right),
$$

and $\left(t_{2 k}, t_{2 k+1}\right)$ is an optimal saddle escaping from $\pi\left(t_{2 k-1}, D \backslash G\right)$ for all $k$ in $\{1, \ldots, h\}$. Then $\left(t_{0}, \ldots, t_{2 h+1}\right)$ may be seen as a portion of a saddle path of $\mathcal{S}$. Since $\left(t_{2 k}, t_{2 k+1}\right)$ is an optimal saddle escaping from $\pi\left(t_{2 k-1}, D \backslash G\right)$ we have

$$
\sum_{k=0}^{h}\left(W\left(t_{2 k}\right)+V\left(t_{2 k}, t_{2 k+1}\right)-H\left(\pi\left(t_{2 k}, D \backslash G\right)\right)-W\left(\pi\left(t_{2 k}, D \backslash G\right)\right)\right)=0 .
$$

Now $\left(s_{0}, s_{1}, t_{0}, \ldots, t_{2 h+1}, s_{2}, \ldots, s_{2 r+1}\right)$ is in the saddle path set $\mathcal{S}$ and has the same $V_{x}$-cost as the initial saddle path $\left(s_{0}, \ldots, s_{2 r+1}\right)$. As a consequence, $V_{x}$ is not in general a good rate function (its level sets are not compact) and with $V_{x}$ we won't be able to control the length of the saddle path. What we have to do is to get rid of sequences like $\left(t_{0}, \ldots, t_{2 h+1}\right)$ by considering only the relevant jumps between cycles of $\mathcal{M}(D \backslash G)$.

\section{THE PRUNED SADDLE PATH}

The pruned saddle path is to the saddle path what the pruned cycle path is to the cycle path.

DEFinition 8.1. (pruned saddle path) We define recursively a sequence of random times and points:

$$
\begin{array}{lcc}
\bar{\tau}_{0}=\theta(G, \tau(D))+1, & \bar{s}_{0}=X_{\bar{\tau}_{0}-1}, & \bar{s}_{1}=X_{\bar{\tau}_{0}}, \\
\bar{\tau}_{1}=\tau\left(\bar{\pi}\left(\bar{s}_{1}, D \backslash G\right), \bar{\tau}_{0}\right), & \bar{s}_{2}=X_{\bar{\tau}_{1}-1}, & \bar{s}_{3}=X_{\bar{\tau}_{1}}, \\
\quad \vdots & \vdots & \vdots \\
\bar{\tau}_{k}=\tau\left(\bar{\pi}\left(\bar{s}_{2 k-1}, D \backslash G\right), \bar{\tau}_{k-1}\right), & \bar{s}_{2 k}=X_{\bar{\tau}_{k}-1}, & \bar{s}_{2 k+1}=X_{\bar{\tau}_{k}}, \\
\vdots & \vdots & \vdots \\
\bar{\tau}_{r}=\tau(D), & \bar{s}_{2 r}=X_{\bar{\tau}_{r}-1}, & \bar{s}_{2 r+1}=X_{\bar{\tau}_{r}} .
\end{array}
$$

The sequence $\left(\bar{s}_{0}, \ldots, \bar{s}_{2 r+1}\right)$ is called the pruned saddle path of $\left(X_{n}\right)$ relative to $D, G$ and is denoted by $\overline{\mathcal{S}}(X, D, G)$. The pruned saddle path space relative to $D, G$ starting at $x$ is the set

$$
\begin{aligned}
& \overline{\mathcal{S}}(D, G)=\left\{\left(\bar{s}_{0}, \ldots, \bar{s}_{2 r+1}\right): \bar{s}_{0} \in G, \bar{s}_{2 r+1} \in D^{c}\right. \\
& \forall k \in\{1, \ldots, r\}, \bar{s}_{2 k-1} \in D \backslash G, \bar{s}_{2 k} \in \bar{\pi}\left(\bar{s}_{2 k-1}, D \backslash G\right), V\left(\bar{s}_{2 k}, \bar{s}_{2 k+1}\right)<\infty \\
& \left.\bar{s}_{2 k+1} \in B\left(\bar{\pi}\left(\bar{s}_{2 k-1}, D \backslash G\right)\right)\right\} .
\end{aligned}
$$


We define a cost function $\bar{V}_{x}(D, G)$ on the space $\mathcal{S}(D, G)$ by

$$
\begin{aligned}
& \bar{V}_{x}(D, G)\left(\bar{s}_{0}, \ldots, \bar{s}_{2 r+1}\right)=W_{D}\left(x, \bar{s}_{0}\right)+V\left(\bar{s}_{0}, \bar{s}_{1}\right) \\
& \quad+\sum_{k=1}^{r}\left(W\left(\bar{s}_{2 k}\right)+V\left(\bar{s}_{2 k}, \bar{s}_{2 k+1}\right)-H\left(\bar{\pi}\left(\bar{s}_{2 k}, D \backslash G\right)\right)-W\left(\bar{\pi}\left(\bar{s}_{2 k}, D \backslash G\right)\right)\right) .
\end{aligned}
$$

Notice that the pruned saddle path of $\left(X_{n}\right)$ is a subsequence of the saddle path of $\left(X_{n}\right)$ : it is obtained by deleting the jumps between equivalent cycles of $\mathcal{M}(D \backslash G)$. These removed jumps have a null cost so that the $V_{x^{-}}$ cost of a saddle path and the $\bar{V}_{x}$-cost of the corresponding pruned saddle path are equal i.e. we have $V_{x}(\mathcal{S}(X))=\bar{V}_{x}(\overline{\mathcal{S}}(X))$.

TheOREM 8.2. (estimation of the probability of a pruned saddle path) There exists a positive constant $K_{7}$ (depending only on the cardinality of $D \backslash$ $G)$ such that for any pruned saddle path $\left(\bar{s}_{0}, \ldots, \bar{s}_{2 r+1}\right)$ in $\overline{\mathcal{S}}(D \backslash G)$ and any $\beta$ we have

$$
\begin{aligned}
\left(K_{7}^{-1} a(\beta)^{K_{7}}\right)^{r+1} \exp -\beta \bar{V}_{x}\left(\bar{s}_{0}, \ldots, \bar{s}_{2 r+1}\right) & \\
\leq & P_{\beta}\left(\overline{\mathcal{S}}(X)=\left(\bar{s}_{0}, \ldots, \bar{s}_{2 r+1}\right) / X_{0}=x\right) \leq \\
& \left(K_{7} a(\beta)^{-K_{7}}\right)^{r+1} \exp -\beta \bar{V}_{x}\left(\bar{s}_{0}, \ldots, \bar{s}_{2 r+1}\right) .
\end{aligned}
$$

The proof is of the same kind as for theorem 7.4. The only difference lies in the equivalent form of lemma 7.2 , that we state now.

LEMMA 8.3. (inner and outer exit points from a set of equivalent cycles) There exists a positive constant $K_{8}$ such that for any cycle $\bar{\pi}, x, y \in \bar{\pi}$ and $z \in B(\bar{\pi})$,

$$
\begin{aligned}
K_{8}^{-1} a(\beta)^{K_{8}} \exp -\beta(W(y)+V(y, z)-H(\bar{\pi})-W(\bar{\pi})) \\
\leq \quad P_{\beta}\left(X_{\tau(\bar{\pi})}=z, X_{\tau(\bar{\pi})-1}=y / X_{0}=x\right) \leq \\
\quad K_{8} a(\beta)^{-K_{8}} \exp -\beta(W(y)+V(y, z)-H(\bar{\pi})-W(\bar{\pi})) .
\end{aligned}
$$

Proof. The proof follows the line of the proof of lemma 7.2. Here the quantity characterizing the rate of decrease of the event under consideration (i.e. the exit from the set $\bar{\pi}$ through the arrow $(y, z))$ is $W_{\bar{\pi}}(x, y)+V(y, z)$. Application of lemma 4.7 yields $W_{\bar{\pi}}(x, y)=C_{\bar{\pi} \backslash\{y\}}(x, y)-C_{\bar{\pi} \backslash\{y\}}\left(y, \bar{\pi}^{c}\right)$. Since $x$ and $y$ are in $\bar{\pi}$, we have by the definition of $\bar{\pi}$ combined with theorem 5.5 that the cost $C_{\bar{\pi} \backslash\{y\}}(x, y)$ is null. Next, proposition 4.14 yields

$$
C_{\bar{\pi} \backslash\{y\}}\left(y, \bar{\pi}^{c}\right)=H(\pi(y, \bar{\pi}))+W(\pi(y, \bar{\pi}))-W(y)
$$

(where we recall that $\pi(y, \bar{\pi})$ is the greatest cycle included in $\bar{\pi}$ which contains $y)$. Anyway the quantity $H(\pi)+W(\pi)$ is the same for all the maximal subcycles of $\bar{\pi}$ and coincides with $H(\bar{\pi})+W(\bar{\pi})$. Thus $W_{\bar{\pi}}(x, y)+V(y, z)=$ $W(y)+V(y, z)-H(\bar{\pi})-W(\bar{\pi})$.

Let $\bar{\Theta}$ be the map from $\overline{\mathcal{S}}(D, G)$ to $\bar{\Psi}(D, G)$ defined by

$$
\bar{\Theta}\left(\bar{s}_{0}, \ldots, \bar{s}_{2 r+1}\right)=\left(\left\{\bar{s}_{0}\right\}, \bar{\pi}\left(\bar{s}_{1}, D \backslash G\right), \ldots, \bar{\pi}\left(\bar{s}_{2 r-1}, D \backslash G\right),\left\{\bar{s}_{2 r+1}\right\}\right) .
$$


Let $\left(\bar{s}_{0}, \ldots, \bar{s}_{2 r+1}\right)$ in $\overline{\mathcal{S}}(D, G)$ and $\left(\bar{\pi}_{-1}, \ldots, \bar{\pi}_{r}\right)$ in $\bar{\Psi}(D, G)$ be such that

$$
\bar{\Theta}\left(\bar{s}_{0}, \ldots, \bar{s}_{2 r+1}\right)=\left(\bar{\pi}_{-1}, \ldots, \bar{\pi}_{r}\right) .
$$

We have the inclusion

$$
\left\{\overline{\mathcal{S}}(X)=\left(\bar{s}_{0}, \ldots, \bar{s}_{2 r+1}\right)\right\} \subset\left\{\bar{\pi}(X)=\left(\bar{\pi}_{-1}, \ldots, \bar{\pi}_{r}\right)\right\}
$$

Theorems 6.2 and 8.2 then imply that $\bar{V}_{x}\left(\bar{s}_{0}, \ldots, \bar{s}_{2 r+1}\right) \geq \bar{V}_{x}\left(\bar{\pi}_{-1}, \ldots, \bar{\pi}_{r}\right)$. As a consequence, the conclusions of corollaries 6.4 and 6.5 are still valid for the rate function $\bar{V}_{x}$ on the space $\overline{\mathcal{S}}$. We restate them in the next two corollaries.

Corollary 8.4. The cost $\bar{V}_{x}$ gives a control on the length of the pruned saddle path i.e.

$\forall \lambda \quad \exists R(\lambda) \quad \forall r \geq R(\lambda) \quad \forall\left(\bar{s}_{0}, \ldots, \bar{s}_{2 r+1}\right) \in \overline{\mathcal{S}}(D \backslash G)$

$$
\bar{V}_{x}\left(\bar{s}_{0}, \ldots, \bar{s}_{2 r+1}\right) \geq \lambda .
$$

Corollary 8.5. The cost function $\bar{V}_{x}$ is a good rate function on $\overline{\mathcal{S}}$ i.e. its level sets are compact (finite in our situation).

The length of the saddle path $\mathcal{S}(X)$ (i.e. the number of jumps it involves) will be denoted by $|\mathcal{S}(X)|$ in the sequel. For instance $\left|\left(s_{0}, \ldots, s_{2 r+1}\right)\right|=r$. Corollary 8.6. There exist a positive constant $K_{9}$ and a function $h$ : $\mathbb{N} \rightarrow \mathbb{R}_{+}$such that $\lim _{+\infty} h=+\infty$ and

$$
P_{\beta}\left(|\overline{\mathcal{S}}(X)| \geq R / X_{0}=x\right) \leq\left(K_{9} a(\beta)^{-K_{9}}\right)^{R} \exp -\beta h(R) .
$$

Proof. This is a straightforward consequence of the equality $|\overline{\mathcal{S}}(X)|=|\bar{\pi}(X)|$ and of proposition 6.6 .

Theorem 8.7. (large deviations upper bound) For any positive $\lambda$, there exists a positive constant $K_{10}$ such that for all $\beta$

$$
P_{\beta}\left(V_{x}(\mathcal{S}(X)) \geq \lambda / X_{0}=x\right) \leq K_{10} a(\beta)^{-K_{10}} \exp -\beta \lambda .
$$

Proof. We decompose the event $\left\{V_{x}(\mathcal{S}(X)) \geq \lambda\right\}$ according to the length of the associated pruned saddle path. Let $R$ be such that $h(R)>\lambda$. We have

$P_{\beta}\left(V_{x}(\mathcal{S}(X)) \geq \lambda / X_{0}=x\right)=P_{\beta}\left(\bar{V}_{x}(\overline{\mathcal{S}}(X)) \geq \lambda / X_{0}=x\right)$

$=P_{\beta}\left(\bar{V}_{x}(\overline{\mathcal{S}}(X)) \geq \lambda,|\overline{\mathcal{S}}(X)| \geq R / X_{0}=x\right)+$

$$
P_{\beta}\left(\bar{V}_{x}(\overline{\mathcal{S}}(X)) \geq \lambda,|\overline{\mathcal{S}}(X)|<R / X_{0}=x\right) .
$$

The first term in the sum is controlled by corollary 8.6. The second term is equal to

$$
\sum P_{\beta}\left(\overline{\mathcal{S}}(X)=\left(\bar{s}_{0}, \ldots, \bar{s}_{2 r+1}\right) / X_{0}=x\right)
$$


where the sum runs over all pruned saddle paths $\left(\bar{s}_{0}, \ldots, \bar{s}_{2 r+1}\right)$ satisfying the conditions $r<R, \bar{V}_{x}\left(\bar{s}_{0}, \ldots, \bar{s}_{2 r+1}\right) \geq \lambda$. For such a path we have by theorem 8.2

$$
P_{\beta}\left(\overline{\mathcal{S}}(X)=\left(\bar{s}_{0}, \ldots, \bar{s}_{2 r+1}\right) / X_{0}=x\right) \leq\left(K_{7} a(\beta)^{-K_{7}}\right)^{r+1} \exp -\beta \lambda
$$

and there is only a finite number of such terms (the length of the paths being bounded). The desired upper bound follows easily.

We have just proved that the law of the pruned saddle path satisfies a large deviation principle with the good rate function $\bar{V}_{x}$. As a consequence, as $\beta$ goes to infinity, this law concentrates on the paths of null $V_{x}$-cost.

Proposition 8.8. (concentration of the law of the saddle path)

$$
\lim _{\beta \rightarrow \infty} P_{\beta}\left(V_{x}(\mathcal{S}(X))=0 / X_{0}=x\right)=1 .
$$

We finally give a criterion to decide whether a saddle path has a positive asymptotic probability to occur.

Proposition 8.9. Assume that $\liminf _{\beta \rightarrow \infty} a(\beta)>0$. Then

$$
\begin{aligned}
\liminf _{\beta \rightarrow \infty} P_{\beta}\left(\mathcal{S}(X)=\left(s_{0}, \ldots, s_{2 r+1}\right) / X_{0}=x\right) & >0 \\
& \Longleftrightarrow V_{x}\left(s_{0}, \ldots, s_{2 r+1}\right)=0 .
\end{aligned}
$$

\section{The SADDLE PATHS OF NULL $V_{x}-\mathrm{COST}$}

This section is devoted to the investigation of the structure of the saddle paths having a null $V_{x}$-cost.

Theorem 9.1. (characterization of the saddle paths of null $V_{x}-\cos$ ) Let $s=\left(s_{0}, \ldots, s_{2 r+1}\right)$ be a saddle path in $\mathcal{S}(D, G)$. Let $r_{*}=\min \left\{k: s_{2 k+1} \notin\right.$ $\left.\pi\left(s_{0}, D\right)\right\}$. The saddle path $s$ has a null $V_{x}-$ cost if and only if

- $C_{D \backslash s_{0}}\left(x, s_{0}\right)=0$,

- $V\left(s_{0}, s_{1}\right)+$

$$
\begin{array}{r}
\sum_{1 \leq k \leq r_{*}}\left(W\left(s_{2 k}\right)+V\left(s_{2 k}, s_{2 k+1}\right)-H\left(\pi\left(s_{2 k}, D \backslash G\right)\right)-W\left(\pi\left(s_{2 k}, D \backslash G\right)\right)\right) \\
=H\left(\pi\left(s_{0}, D\right)\right)+W\left(\pi\left(s_{0}, D\right)\right)-W\left(s_{0}\right)
\end{array}
$$

- $\forall k, \quad r_{*}<k \leq r, \quad\left(s_{2 k}, s_{2 k+1}\right)$ is an optimal saddle escaping from the cycle $\pi\left(s_{2 k-1}, D \backslash G\right)$.

REMARK. The second property is equivalent to saying that $s_{2 r_{*}+1}$ is in the principal boundary of $\pi\left(s_{0}, D\right)$ and that the sequence $\left(s_{0}, \ldots, s_{2 r_{*}+1}\right)$ is a saddle path of null $V_{s_{0}}-$ cost of $\Psi\left(\pi\left(s_{0}, D\right), G\right)$.

REMARK. We recall that the third property says that (see definition 7.5 )

$$
\begin{gathered}
\forall k \in\left\{r_{*}+1, \ldots, r\right\}, \quad s_{2 k} \in \pi\left(s_{2 k-1}, D \backslash G\right), \quad s_{2 k+1} \in \widetilde{B}\left(\pi\left(s_{2 k-1}, D \backslash G\right)\right), \\
W\left(s_{2 k}\right)+V\left(s_{2 k}, s_{2 k+1}\right)=W\left(\pi\left(s_{2 k-1}, D \backslash G\right)\right)+H\left(\pi\left(s_{2 k-1}, D \backslash G\right)\right) .
\end{gathered}
$$


Proof. We have the inequalities

$$
\begin{aligned}
& V_{x}\left(s_{0}, \ldots, s_{2 r+1}\right) \geq W_{D}\left(x, s_{0}\right)+V\left(s_{0}, s_{1}\right)+ \\
& \sum_{1 \leq k \leq r_{*}}\left(W\left(s_{2 k}\right)+V\left(s_{2 k}, s_{2 k+1}\right)-H\left(\pi\left(s_{2 k}, D \backslash G\right)\right)-W\left(\pi\left(s_{2 k}, D \backslash G\right)\right)\right) \\
& (\text { Corollary } 7.3) \geq W_{D}\left(x, s_{0}\right)+V\left(s_{0}, s_{1}\right) \\
& +\sum_{1 \leq k \leq r_{*}} C_{\pi\left(s_{2 k}, D \backslash G\right)}\left(s_{2 k}, s_{2 k+1}\right) \\
& \left(\begin{array}{c}
\text { Lemma 4.3 } \\
\text { Proposition 4.4 }
\end{array}\right) \geq W_{D}\left(x, s_{0}\right)+C_{\pi\left(s_{0}, D\right) \backslash G}\left(s_{0}, s_{2 r_{*}+1}\right) \\
& \text { (Lemma 4.7) }=C_{D \backslash\left\{s_{0}\right\}}\left(x, s_{0}\right)-C_{D \backslash\left\{s_{0}\right\}}\left(s_{0}, D^{c}\right) \\
& +C_{\pi\left(s_{0}, D\right) \backslash G}\left(s_{0}, s_{2 r_{*}+1}\right) \\
& \text { (Corollary 4.15) }=C_{D \backslash\left\{s_{0}\right\}}\left(x, s_{0}\right)-C_{\pi\left(s_{0}, D\right) \backslash\left\{s_{0}\right\}}\left(s_{0}, \pi\left(s_{0}, D\right)^{c}\right) \\
& +C_{\pi\left(s_{0}, D\right) \backslash G}\left(s_{0}, s_{2 r_{*}+1}\right) \\
& \geq C_{D \backslash\left\{s_{0}\right\}}\left(x, s_{0}\right) \geq 0 .
\end{aligned}
$$

The first inequality is obtained by truncating the sum in the definition of the cost $V_{x}$. The third inequality relies on the following facts:

$V\left(s_{0}, s_{1}\right) \geq C_{\pi\left(s_{0}, D\right) \backslash G}\left(s_{0}, s_{1}\right), \quad \pi\left(s_{2 k}, D \backslash G\right) \subset \pi\left(s_{0}, D\right) \backslash G, \quad 1 \leq k \leq r_{*}$.

Thus, for the saddle path to have a null cost, all these successive inequalities have to be equalities. That is, we must have $C_{D \backslash\left\{s_{0}\right\}}\left(x, s_{0}\right)=0$, the quantity $V\left(s_{0}, s_{1}\right)+$

$$
\sum_{1 \leq k \leq r_{*}}\left(W\left(s_{2 k}\right)+V\left(s_{2 k}, s_{2 k+1}\right)-H\left(\pi\left(s_{2 k}, D \backslash G\right)\right)-W\left(\pi\left(s_{2 k}, D \backslash G\right)\right)\right)
$$

must be equal to $H\left(\pi\left(s_{0}, D\right)\right)+W\left(\pi\left(s_{0}, D\right)\right)-W\left(s_{0}\right)$ and for all $k$ in $\left\{r_{*}+1, \ldots, r\right\}$, we must have $W\left(s_{2 k}\right)+V\left(s_{2 k}, s_{2 k+1}\right)-H\left(\pi\left(s_{2 k}, D \backslash G\right)\right)-$ $W\left(\pi\left(s_{2 k}, D \backslash G\right)\right)=0$, this last condition being equivalent to saying that $\left(s_{2 k}, s_{2 k+1}\right)$ is an optimal saddle escaping from the cycle $\pi\left(s_{2 k}, D \backslash G\right)$.

Conversely, if these three conditions are satisfied, we check directly that the $V_{x}$-cost of the saddle path is null.

Definition 9.2. Let $A$ and $B$ be two disjoint subsets of $E$. We define $\mathcal{V}(A, B)$, the set of points from which $A$ is visited before $B$, by

$$
\mathcal{V}(A, B)=\left\{x \in E: C_{A^{c} \cap B^{c}}(x, B)>0\right\} .
$$

We define $\mathcal{R}(A, B)$, the set of ridge points between $A$ and $B$, by

$$
\mathcal{R}(A, B)=\left\{x \in E: C_{A^{c} \cap B^{c}}(x, B)=C_{A^{c} \cap B^{c}}(x, A)=0\right\} .
$$

The probabilistic counterpart of this definition is the following: a point $x$ is in $\mathcal{V}(A, B)$ if and only if

$$
\lim _{\beta \rightarrow \infty}-\frac{1}{\beta} \ln P_{\beta}\left(\tau\left(B^{c}\right)<\tau\left(A^{c}\right) / X_{0}=x\right)>0 ;
$$


a point $x$ is in $\mathcal{R}(A, B)$ if and only if

$$
\begin{aligned}
& \lim _{\beta \rightarrow \infty}-\frac{1}{\beta} \ln P_{\beta}\left(\tau\left(B^{c}\right)<\tau\left(A^{c}\right) / X_{0}=x\right)=0, \\
& \lim _{\beta \rightarrow \infty}-\frac{1}{\beta} \ln P_{\beta}\left(\tau\left(B^{c}\right)>\tau\left(A^{c}\right) / X_{0}=x\right)=0 .
\end{aligned}
$$

Remark. Notice that for any point of $E$, we have $C_{A^{c} \cap B^{c}}(x, A \cup B)=0$ so that at least one of the two costs $C_{A^{c} \cap B^{c}}(x, A), C_{A^{c} \cap B^{c}}(x, B)$ is null. Henceforth,

$$
\begin{aligned}
& C_{A^{c} \cap B^{c}}(x, A)>0 \quad \Longrightarrow \quad C_{A^{c} \cap B^{c}}(x, B)=0, \\
& C_{A^{c} \cap B^{c}}(x, B)>0 \quad \Longrightarrow \quad C_{A^{c} \cap B^{c}}(x, A)=0 .
\end{aligned}
$$

As a consequence, the three sets $\mathcal{V}(A, B), \mathcal{R}(A, B), \mathcal{V}(B, A)$ form a partition of $E$.

Proposition 9.3. Let $s=\left(s_{0}, \ldots, s_{2 r+1}\right)$ be a saddle path in $\mathcal{S}(D, G)$ of null $V_{x}$-cost. This saddle path visits successively the three sets $\mathcal{V}\left(G, D^{c}\right)$, $\mathcal{R}\left(G, D^{c}\right), \mathcal{V}\left(D^{c}, G\right)$ : if we let $r_{*}=\min \left\{k: s_{2 k+1} \notin \mathcal{V}\left(G, D^{c}\right)\right\}$ and $r^{*}=$ $\min \left\{k \geq r_{*}: s_{2 k+1} \notin \mathcal{R}\left(G, D^{c}\right)\right\}$, then

$$
\begin{aligned}
\forall k, \quad 0 \leq k \leq 2 r_{*}, & s_{k} & \in \mathcal{V}\left(G, D^{c}\right), \\
\forall k, \quad 2 r_{*}<k \leq 2 r^{*}, & s_{k} & \in \mathcal{R}\left(G, D^{c}\right), \\
\forall k, \quad 2 r^{*}<k \leq 2 r+1, & s_{k} & \in \mathcal{V}\left(D^{c}, G\right) .
\end{aligned}
$$

In addition the index $r_{*}$ coincides with $\min \left\{k: s_{2 k+1} \notin \pi\left(s_{0}, D\right)\right\}$. Thus a saddle path of null $V_{x}$-cost may be decomposed into three parts: an ascending part $\left(s_{k}, 0 \leq k \leq 2 r_{*}\right)$, a ridge part $\left(s_{k}, 2 r_{*}<k \leq 2 r^{*}\right)$ and a descending part $\left(s_{k}, 2 r^{*}<k \leq 2 r+1\right)$.

Proof. We first prove that the two indices $r_{*}^{1}=\min \left\{k: s_{2 k+1} \notin \pi\left(s_{0}, D\right)\right\}$ and $r_{*}^{2}=\min \left\{k: s_{2 k+1} \notin \mathcal{V}\left(G, D^{c}\right)\right\}$ are equal. We have $\pi\left(s_{0}, D\right) \subset$ $\mathcal{V}\left(G, D^{c}\right)$ so that $r_{*}^{1} \leq r_{*}^{2}$. Theorem 9.1 shows that $C_{D \backslash G}\left(s_{2 r_{*}^{1}+1}, D^{c}\right)=0$ so that $s_{2 r_{*}^{1}+1}$ is not in $\mathcal{V}\left(G, D^{c}\right)$ and $r_{*}^{1} \geq r_{*}^{2}$. Thus $r_{*}=r_{*}^{1}=r_{*}^{2}$.

That the points $s_{k}, 0 \leq k \leq 2 r_{*}$, are in $\mathcal{V}\left(G, D^{c}\right)$ is straightforward; in fact they all belong to $\pi\left(s_{0}, D\right)$ (remember that $s_{2 k} \in \pi\left(s_{2 k-1}, D \backslash G\right)$ ). Theorem 9.1 shows that all the subsequent points of the saddle path satisfy $C_{D \backslash G}\left(s_{k}, D^{c}\right)=0$ so that they are not in $\mathcal{V}\left(G, D^{c}\right)$. The definition of $r^{*}$ implies that $s_{k} \in \mathcal{R}\left(G, D^{c}\right)$ for $2 r_{*}<k \leq 2 r^{*}$. Finally, theorem 9.1 shows that for $k>2 r^{*}$, we have $C_{D \backslash G}\left(s_{2 r^{*}+1}, s_{k}\right)=0$. Each point $z$ such that $C_{D \backslash G}\left(s_{2 r^{*}+1}, z\right)=0$ is such that $C_{D \backslash G}(z, G)>0$ and is thus in $\mathcal{V}\left(D^{c}, G\right)$ (otherwise we would have $C_{D \backslash G}\left(s_{2 r^{*}+1}, G\right)=0$ ). Therefore the points $\left(s_{k}, 2 r^{*}<k \leq 2 r+1\right)$ are in $\mathcal{V}\left(D^{c}, G\right)$.

DeFinition 9.4. (global saddles) Let $s=\left(s_{0}, \ldots, s_{2 r+1}\right)$ be a saddle path in $\mathcal{S}(D, G)$ of null $V_{s_{0}}$-cost. The global saddles of this saddle path are the saddles $\left(\left(s_{2 k}, s_{2 k+1}\right), r_{*} \leq k \leq r^{*}\right)$. The set of the global saddles between $G$ and $D^{c}$ is the union of the global saddles of all the saddle paths of $\mathcal{S}(D, G)$ of null cost. 
Definition 9.5. (global saddle points) A point $y$ is a global saddle point between $G$ and $D^{c}$ if there exists a global saddle between $G$ and $D^{c}$ entering the cycle $\pi(y, D \backslash G)$ and a global saddle between $G$ and $D^{c}$ escaping from the cycle $\pi(y, D \backslash G)$.

REMARK. Equivalently, the global saddle points are the ridge points between $G$ and $D^{c}$ which may be visited with a non exponentially vanishing probability during the last excursion from $G$ before leaving $D$.

The task of determining the set of all the saddle paths of null $V_{x}-$ cost can be performed in the following way:

i) first find the points $s$ of $G$ such that $C_{D}(x, s)=0$;

ii) for each such point $s$, find the points $s^{\prime}$ of the principal boundary of the cycle $\pi(s, D)$;

iii) for each such point $s^{\prime}$ in $\widetilde{B}(\pi(s, D))$, determine all the sequences of cycles $\pi_{0}, \ldots, \pi_{r}$ in $\mathcal{M}(D \backslash G)$ such that $s^{\prime} \in \pi_{0}, \widetilde{B}\left(\pi_{k}\right) \cap \pi_{k+1} \neq \emptyset$ for $k$ in $\{0, \ldots, r-1\}, \widetilde{B}\left(\pi_{r}\right) \cap D^{c} \neq \emptyset$;

iv) for each such cycle path $\pi_{0}, \ldots, \pi_{r}$ determine all the saddle paths $\left(s_{0}, \ldots, s_{2 r+1}\right)$ such that $s_{0} \in \pi_{0}, s_{2 r+1} \in D^{c},\left(s_{2 k-1}, s_{2 k}\right) \in \pi_{k} \times \pi_{k}$ for $k$ in $\{1, \ldots, r\}$, and $\left(s_{2 k}, s_{2 k+1}\right)$ is an optimal saddle escaping from $\pi_{k}$ for all $k$ in $\{0, \ldots, r\}$;

v) finally determine all the saddle paths $\left(s_{0}^{\prime}, \ldots, s_{2 r^{\prime}+1}^{\prime}\right)$ in $\Psi(\pi(s, D), G)$ starting at $s_{0}^{\prime} \in \pi(s, D) \cap G$, ending at $s_{2 r^{\prime}+1}^{\prime}=s^{\prime}$ and such that the cost

$$
\sum_{1 \leq k \leq r^{\prime}}^{V\left(s_{0}^{\prime}, s_{1}^{\prime}\right)+}\left(W\left(s_{2 k}^{\prime}\right)+V\left(s_{2 k}^{\prime}, s_{2 k+1}^{\prime}\right)-H\left(\pi\left(s_{2 k}^{\prime}, D \backslash G\right)\right)-W\left(\pi\left(s_{2 k}^{\prime}, D \backslash G\right)\right)\right)
$$

is equal to $H\left(\pi\left(s_{0}^{\prime}, D\right)\right)+W\left(\pi\left(s_{0}^{\prime}, D\right)\right)-W\left(s_{0}^{\prime}\right)$;

vi) the set of all the saddle paths of null $V_{x}-$ cost passing through $s^{\prime}$ is the set of the saddle paths $\left(s_{0}^{\prime}, \ldots, s_{2 r^{\prime}+1}^{\prime}, s_{0}, s_{1}, \ldots, s_{2 r+1}\right)$ obtained by gluing together two saddle paths obtained at step $i v$ ) and $v$ ).

Remark that step iii) may fail: it may happen that there is no cycle path of null cost in $\mathcal{M}(D \backslash G)$ starting at $s^{\prime}$ and escaping from $D$. In that case, there is no saddle path of null cost passing through $s^{\prime}$ and it is not necessary to perform step $v$ ) (this is the reason why we propose to do step $v$ ) at the end). Such a point $s^{\prime}$ leads to a dead-end. What we do at step iii) is to look at all the cycles we can reach from $s^{\prime}$ in the graph over $\mathcal{M}(D \backslash G)$ defined by the relation

$$
\pi_{1} \rightarrow \pi_{2} \quad \Longleftrightarrow \quad \widetilde{B}\left(\pi_{1}\right) \cap \pi_{2} \neq \emptyset \quad \Longleftrightarrow \quad C_{\pi_{1}}\left(\pi_{1}, \pi_{2}\right)=0
$$

i.e. we determine the orbit of the cycle $\pi\left(s^{\prime}, D \backslash G\right)$ in this graph (the minimal stable subgraph containing $\pi\left(s^{\prime}, D \backslash G\right)$ ). The good cycle paths are the cycle paths included in this graph which end at a cycle whose principal boundary contains points of $D^{c}$.

Depending on the situation, one might also determine first the pruned cycle paths of null cost and then search for the whole cycle paths.

Saddle paths corresponding to step $v$ ) always exist: in fact, we have $\pi(s, D) \cap$ $G \neq \emptyset$ and thus for all $s_{0}$ in $\pi(s, D) \cap G$, the cost $C_{D}\left(x, s_{0}\right)$ is null. We 
consider for instance a saddle path of null $V_{s}(\pi(s, D),\{s\})$-cost starting at $s$ and escaping from $\pi(s, D)$ at $s^{\prime}$ (such a saddle path exists since $s^{\prime}$ is in the principal boundary of $\pi(s, D))$. We choose for $s_{0}^{\prime}$ the last point of the saddle path belonging to $G$. The portion of the saddle path after $s_{0}^{\prime}$ satisfies the requirements.

\section{TIME SPENT IN EACH STATE OF THE EXIT PATH}

We now study the times $\tau_{1}-\tau_{0}, \ldots, \tau_{r+1}-\tau_{r}$ between the jumps along the saddle path as well as the times the process spends in the points of the cycle path, knowing that the Markov chain follows a fixed saddle path. The key lies in the following Wentzell-Freidlin type formulas.

LEMma 10.1. (conditional expected number of visits before exit) For an arbitrary set $W$, two points $x, z$ in $W^{c}$ and $y$ in $W$, we have

$$
\begin{aligned}
\sum_{n=0}^{\infty} P_{\beta}\left(X_{n}=z, \tau\left(W^{c}\right)>n / X_{0}=x, X_{\tau\left(W^{c}\right)}=y\right)= & \frac{\sum_{g_{1} \in G_{x, z}(W \cup\{z\})} p_{\beta}\left(g_{1}\right) \sum_{g_{2} \in G_{z, y}(W)} p_{\beta}\left(g_{2}\right)}{\sum_{g_{1} \in G(W)} p_{\beta}\left(g_{1}\right) \sum_{g_{2} \in G_{x, y}(W)} p_{\beta}\left(g_{2}\right)}
\end{aligned}
$$

Proof. We just write

$$
\begin{aligned}
\sum_{n=0}^{\infty} P_{\beta}\left(X_{n}=z, \tau\left(W^{c}\right)>n / X_{0}=x, X_{\tau\left(W^{c}\right)}=y\right) \\
=\sum_{n=0}^{\infty} \frac{P_{\beta}\left(\tau\left(W^{c}\right)>n, X_{\tau\left(W^{c}\right)}=y, X_{n}=z / X_{0}=x\right)}{P_{\beta}\left(X_{\tau\left(W^{c}\right)}=y / X_{0}=x\right)} \\
=\sum_{n=0}^{\infty} \frac{P_{\beta}\left(\tau\left(W^{c}\right)>n, X_{n}=z / X_{0}=x\right) P_{\beta}\left(X_{\tau\left(W^{c}\right)}=y / X_{0}=z\right)}{P_{\beta}\left(X_{\tau\left(W^{c}\right)}=y / X_{0}=x\right)}
\end{aligned}
$$

and the result follows by applying lemmas 3.5 and 3.7 .

Lemma 10.2. (conditional expected exit time) For an arbitrary set $W, x$ in $W^{c}$ and $y$ in $W$, we have

$$
E_{\beta}\left(\tau\left(W^{c}\right) / X_{0}=x, X_{\tau\left(W^{c}\right)}=y\right)=\frac{\sum_{z \in W^{c}} \sum_{g_{1} \in G_{x, z}(W \cup\{z\})} p_{\beta}\left(g_{1}\right) \sum_{g_{2} \in G_{z, y}(W)} p_{\beta}\left(g_{2}\right)}{\sum_{g_{1} \in G(W)} p_{\beta}\left(g_{1}\right) \sum_{g_{2} \in G_{x, y}(W)} p_{\beta}\left(g_{2}\right)}
$$

Proof. We either do a proof in the spirit of the proof of lemma 10.1 or we simply write

$$
\begin{aligned}
& E_{\beta}\left(\tau\left(W^{c}\right) / X_{0}=x, X_{\tau\left(W^{c}\right)}=y\right)= \\
& \sum_{z \in W^{c}} \sum_{n=0}^{\infty} P_{\beta}\left(X_{n}=z, \tau\left(W^{c}\right)>n / X_{0}=x, X_{\tau\left(W^{c}\right)}=y\right)
\end{aligned}
$$


and apply the formula proved in lemma 10.1 .

REMARK. It is interesting to notice that for these conditional formulas the graph summations are coupled in the numerator and are independent for the denominator.

Corollary 10.3. (large deviations of the conditional expected number of visits) As $\beta$ goes to infinity,

$$
\frac{1}{\beta} \ln \sum_{n=0}^{\infty} P_{\beta}\left(X_{n}=z, \tau\left(W^{c}\right)>n / X_{0}=x, X_{\tau\left(W^{c}\right)}=y\right)
$$

goes to

$$
\begin{aligned}
\min \left\{V\left(g_{1}\right): g_{1} \in G(W)\right\}+\min \left\{V\left(g_{2}\right): g_{2} \in G_{x, y}(W)\right\}- \\
\\
\quad \min \left\{V\left(g_{1}\right)+V\left(g_{2}\right): g_{1} \in G_{x, z}(W \cup\{z\}), g_{2} \in G_{z, y}(W)\right\} .
\end{aligned}
$$

Corollary 10.4. (large deviations of the expected conditional exit time) As $\beta$ goes to infinity, $\ln E\left(\tau\left(W^{c}\right) / X_{0}=x, X_{\tau\left(W^{c}\right)}=y\right) / \beta$ goes to

$$
\begin{aligned}
& \min \left\{V\left(g_{1}\right): g_{1} \in G(W)\right\}+\min \left\{V\left(g_{2}\right): g_{2} \in G_{x, y}(W)\right\}- \\
& \min \left\{V\left(g_{1}\right)+V\left(g_{2}\right): g_{1} \in G_{x, z}(W \cup\{z\}), g_{2} \in G_{z, y}(W), z \in W^{c}\right\} .
\end{aligned}
$$

In general these quantities do really depend on the conditioning event. However we will only be concerned with the case of a cycle.

Proposition 10.5. For a cycle $\pi$, two points $x, z$ in $\pi$ and $y$ in $B(\pi)$ we have

$$
\begin{aligned}
\lim _{\beta \rightarrow \infty} \frac{1}{\beta} \ln \sum_{n=0}^{\infty} P_{\beta}\left(X_{n}=z, \tau(\pi)>n / X_{0}=x, X_{\tau(\pi)}=\right. & = \\
& W(\pi)+H(\pi)-W(z), \\
\lim _{\beta \rightarrow \infty} \frac{1}{\beta} \ln E\left(\tau(\pi) / X_{0}=x, X_{\tau(\pi)}=y\right) & =H(\pi)
\end{aligned}
$$

that is, the expected number of visits to a point and the exit time are of the same order (on the logarithmic scale) as if there were no condition imposed on the exit point.

Proof. Let $\pi$ be a cycle, let $x$ belong to $\pi$ and let $y$ belong to $B(\pi)$. Since $\pi$ is a cycle, for any $z$ in $\pi$, the minimum $\min \left\{V\left(g_{2}\right): g_{2} \in G_{z, y}\left(\pi^{c}\right)\right\}$ is equal to $\min \left\{V\left(g_{2}\right): g_{2} \in G_{x, y}\left(\pi^{c}\right)\right\}$ (a minimizing graph will have only one arrow exiting from $\pi$ and this arrow will land in $y$ ). The quantity appearing in corollary 10.3 is thus reduced to

$$
\min \left\{V\left(g_{1}\right): g_{1} \in G\left(\pi^{c}\right)\right\}-\min \left\{V\left(g_{1}\right): g_{1} \in G_{x, z}\left(\pi^{c} \cup\{z\}\right)\right\}
$$

which is exactly $C_{\pi \backslash\{z\}}\left(z, \pi^{c}\right)=W(\pi)+H(\pi)-W(z)$ (see proposition 4.14 and the beginning of its proof). This proves the first formula stated in the proposition. Taking the infimum over $z$ in $\pi$ (which corresponds to the 
quantity appearing in corollary 10.4$)$, we obtain $H(\pi)$, proving the second formula.

Proposition 10.6. (estimation of the conditioned number of visits to a state before exit) Let $1(A)$ denote the characteristic function of the event $A$. For any cycle $\pi$, any $x, u$ in $\pi$, any $y$ in $B(\pi)$, for any positive $\epsilon$, the limit

$\lim _{\beta \rightarrow \infty}-\frac{1}{\beta} \ln P_{\beta}\left(\sum_{k=0}^{\tau(\pi)} 1\left(X_{k}=u\right)>\exp -\beta\left(W_{\pi}(u, u)-\epsilon\right) / X_{0}=x, X_{\tau(\pi)}=y\right)$

is infinite, and for any small enough positive $\epsilon$ the limit

$\liminf _{\beta \rightarrow \infty}-\frac{1}{\beta} \ln P_{\beta}\left(\sum_{k=0}^{\tau(\pi)} 1\left(X_{k}=u\right)<\exp -\beta\left(W_{\pi}(u, u)+\epsilon\right) / X_{0}=x, X_{\tau(\pi)}=y\right)$

is larger than $\epsilon$.

Proof. Let us consider the random variable

$$
\phi=\sum_{k=0}^{\tau(\pi)} 1\left(X_{k}=u\right)
$$

and the sequence of stopping times

$$
\begin{aligned}
& \theta_{0}=\inf \left\{n \geq 0: X_{n}=u\right\}, \\
& \theta_{k}=\inf \left\{n>\theta_{k-1}: X_{n}=u\right\}, \text { for } k \geq 1 .
\end{aligned}
$$

For any $N \geq M>0$ we have

$$
\begin{gathered}
P_{\beta}\left(\phi>N, X_{\tau(\pi)}=y / X_{0}=x\right)=P_{\beta}\left(\theta_{N}<\tau(\pi), X_{\tau(\pi)}=y / X_{0}=x\right) \\
=P_{\beta}\left(\theta_{0}<\tau(\pi) / X_{0}=x\right) P_{\beta}\left(\theta_{N}<\tau(\pi) / X_{0}=u\right) P_{\beta}\left(X_{\tau(\pi)}=y / X_{0}=u\right) \\
\leq P_{\beta}\left(\theta_{0}<\tau(\pi) / X_{0}=x\right) P_{\beta}\left(X_{\tau(\pi)}=y / X_{0}=u\right) \\
\times P_{\beta}\left(\theta_{M}<\tau(\pi) / X_{0}=u\right)^{\lfloor N / M\rfloor} \\
\leq P_{\beta}\left(X_{\tau(\pi)}=y / X_{0}=x\right)\left(\frac{E_{\beta}\left(\phi / X_{0}=u\right)}{M}\right)^{\lfloor N / M\rfloor}
\end{gathered}
$$

Let us take $M=\left\lceil e E_{\beta}\left(\phi / X_{0}=u\right)\right\rceil$. We obtain

$$
\begin{aligned}
P_{\beta}\left(\phi>N, X_{\tau(\pi)}=y / X_{0}=x\right) & \leq \\
P_{\beta}\left(X_{\tau(\pi)}\right. & \left.=y / X_{0}=x\right) \exp -\left\lfloor\frac{N}{e E_{\beta}\left(\phi / X_{0}=u\right)+1}\right\rfloor
\end{aligned}
$$

so that

$$
P_{\beta}\left(\phi>N / X_{\tau(\pi)}=y, X_{0}=x\right) \leq \exp -\left\lfloor\frac{N}{e E_{\beta}\left(\phi / X_{0}=u\right)+1}\right\rfloor
$$


from which it is easy to deduce the first equation of proposition 10.6.

We now prove the second inequality. We have

$$
\begin{gathered}
P_{\beta}\left(\phi<N, X_{\tau(\pi)}=y / X_{0}=x\right)=\sum_{k=0}^{N-1} P_{\beta}\left(\phi=k, X_{\tau(\pi)}=y / X_{0}=x\right) \\
=P_{\beta}\left(X_{\tau(\pi \backslash\{u\})}=y / X_{0}=x\right)+ \\
\quad \sum_{k=1}^{N-1} P_{\beta}\left(\theta_{k}<\tau(\pi), X_{\tau(\pi)}=y, \theta_{k+1}>\tau(\pi) / X_{0}=x\right) \\
=P_{\beta}\left(X_{\tau(\pi \backslash\{u\})}=y / X_{0}=x\right)+ \\
\sum_{k=1}^{N-1} P_{\beta}\left(\theta_{k}<\tau(\pi) / X_{0}=x\right) P_{\beta}\left(X_{\tau(\pi \backslash\{u\})}=y / X_{0}=u\right) .
\end{gathered}
$$

Moreover

$$
P_{\beta}\left(X_{\tau(\pi)}=y / X_{0}=u\right)=P_{\beta}\left(X_{\tau(\pi \backslash\{u\})}=y / X_{0}=u\right) E_{\beta}\left(\phi / X_{0}=u\right)
$$

so that

$$
\begin{aligned}
& P_{\beta}\left(\phi<N, X_{\tau(\pi)}=y / X_{0}=x\right) \leq \\
& \quad P_{\beta}\left(X_{\tau(\pi \backslash\{u\})}=y / X_{0}=x\right)+\frac{N-1}{E_{\beta}\left(\phi / X_{0}=u\right)} P_{\beta}\left(X_{\tau(\pi)}=y / X_{0}=u\right)
\end{aligned}
$$

and the second inequality stated in proposition 10.6 will follow from the two following equations:

$$
\begin{gathered}
\lim _{\beta \rightarrow \infty} \frac{P_{\beta}\left(X_{\tau(\pi)}=y / X_{0}=u\right)}{P_{\beta}\left(X_{\tau(\pi)}=y / X_{0}=x\right)}=1, \\
\liminf _{\beta \rightarrow \infty}-\frac{1}{\beta} \ln \frac{P_{\beta}\left(X_{\tau(\pi \backslash\{u\})}=y / X_{0}=x\right)}{P_{\beta}\left(X_{\tau(\pi)}=y / X_{0}=x\right)}>0 .
\end{gathered}
$$

Formula (10.1) follows from the three equations:

$$
\begin{aligned}
& P_{\beta}\left(X_{\tau(\pi)}=y / X_{0}=u\right) \geq P_{\beta}\left(X_{\tau(\pi \backslash\{x\})}=x / X_{0}=u\right) P_{\beta}\left(X_{\tau(\pi)}=y / X_{0}=x\right) \\
& P_{\beta}\left(X_{\tau(\pi)}=y / X_{0}=x\right) \geq P_{\beta}\left(X_{\tau(\pi \backslash\{u\})}=u / X_{0}=x\right) P_{\beta}\left(X_{\tau(\pi)}=y / X_{0}=u\right) \\
& \lim _{\beta \rightarrow \infty} P_{\beta}\left(X_{\tau(\pi \backslash\{u\})}=u / X_{0}=x\right)=\lim _{\beta \rightarrow \infty} P_{\beta}\left(X_{\tau(\pi \backslash\{x\})}=x / X_{0}=u\right)=1 .
\end{aligned}
$$

Let us finally prove formula (10.2). We have

$$
\begin{aligned}
& \frac{P_{\beta}\left(X_{\tau(\pi \backslash\{u\})}=y / X_{0}=x\right)}{P_{\beta}\left(X_{\tau(\pi)}=y / X_{0}=x\right)}= \\
& \quad 1-P_{\beta}\left(X_{\tau(\pi \backslash\{u\})}=u / X_{0}=x\right) \frac{P_{\beta}\left(X_{\tau(\pi)}=y / X_{0}=u\right)}{P_{\beta}\left(X_{\tau(\pi)}=y / X_{0}=x\right)} \\
& \leq 1-\left(1-P_{\beta}\left(X_{\tau(\pi \backslash\{u\})} \neq u / X_{0}=x\right)\right)\left(1-P_{\beta}\left(X_{\tau(\pi \backslash\{x\})} \neq x / X_{0}=u\right)\right) .
\end{aligned}
$$


Moreover, we know that

$$
\begin{aligned}
& \liminf _{\beta \rightarrow \infty}-\frac{1}{\beta} \ln P_{\beta}\left(X_{\tau(\pi \backslash\{u\})} \neq u / X_{0}=x\right)>0, \\
& \liminf _{\beta \rightarrow \infty}-\frac{1}{\beta} \ln P_{\beta}\left(X_{\tau(\pi \backslash\{x\})} \neq x / X_{0}=u\right)>0 .
\end{aligned}
$$

We can thus make an asymptotic expansion in formula (10.3), from which formula (10.2) follows.

Corollary 10.7. For any cycle $\pi$, any $x, u$ in $\pi$, any $y$ in $B(\pi)$, any positive $\epsilon$, the probability

$P_{\beta}\left(\exp -\beta \epsilon \leq \sum_{k=0}^{\tau(\pi)} 1\left(X_{k}=u\right) \exp \beta W_{\pi}(u, u) \leq \exp \beta \epsilon / X_{0}=x, X_{\tau(\pi)}=y\right)$

is going to 1 as $\beta$ goes to infinity.

REMARK. It is clear from our proof that we could get stronger inequalities than those of proposition 10.6 if we strengthen the hypothesis on $a(\beta)$ (for instance $\lim a(\beta)=a>0)$. We leave the details to the reader.

Corollary 10.8. (estimation of the conditioned exit time) For each $\epsilon>0$, each cycle $\pi, x$ in $\pi, y$ in $B(\pi)$, the probability

$$
P_{\beta}\left(\exp \beta(H(\pi)-\epsilon) \leq \tau(\pi) \leq \exp \beta(H(\pi)+\epsilon) / X_{0}=x, X_{\tau(\pi)}=y\right)
$$

is going to 1 as $\beta$ goes to infinity.

Proposition 10.9. For any saddle path $\left(s_{0}, \ldots, s_{2 r+1}\right)$ we have

$$
\begin{aligned}
\lim _{\beta \rightarrow \infty} P_{\beta}\left(\forall k \in\{1, \ldots, r\} \quad \exp \beta\left(H\left(\pi\left(s_{2 k}, D \backslash G\right)\right)-\epsilon\right) \leq \tau_{k+1}-\tau_{k}\right. \\
\leq \exp \beta\left(H\left(\pi\left(s_{2 k}, D \backslash G\right)+\epsilon\right)\right)=1
\end{aligned}
$$

conditionally on the event

$$
\left\{\pi(X)=\left(\left\{s_{0}\right\}, \pi\left(s_{1}, D \backslash G\right), \ldots, \pi\left(s_{2 r-1}, D \backslash G\right),\left\{s_{2 r+1}\right\}\right)\right\} .
$$

REMARK. The conditioning can also be done with respect to the event $\left\{\mathcal{S}(X)=\left(s_{0}, \ldots, s_{2 r+1}\right)\right\}$.

Proof. This result follows from repeated conditioning and applications of the preceding corollary 10.8 .

Proposition 10.10. For any cycle path $\left(\{y\}, \pi_{0}, \ldots, \pi_{r-1},\{z\}\right)$ we have

$$
\begin{aligned}
& \lim _{\beta \rightarrow \infty} P_{\beta}\left(\forall u \in \bigcup_{k=0}^{r-1} \pi_{k}, \quad \exp -\beta\left(W_{D \backslash G}(u, u)+\epsilon\right) \leq \sum_{n=\theta(G, \tau(D))}^{\tau(D)} 1\left(X_{n}=u\right)\right. \\
& \left.\leq \exp -\beta\left(W_{D \backslash G}(u, u)-\epsilon\right) / \pi(X, D, G)=\left(\{y\}, \pi_{0}, \ldots, \pi_{r-1},\{z\}\right)\right)=1 .
\end{aligned}
$$


More precisely, for any $u$ in $\bigcup_{k=0}^{r-1} \pi_{k}$, for any positive $\epsilon$,

$$
\begin{aligned}
\lim _{\beta \rightarrow \infty}-\frac{1}{\beta} \ln P_{\beta}\left(\sum_{n=\theta(G, \tau(D))}^{\tau(D)} 1\left(X_{n}=u\right)>\exp -\beta\left(W_{D \backslash G}(u, u)-\epsilon\right)\right. \\
\left./ \pi(X, D, G)=\left(\{y\}, \pi_{0}, \ldots, \pi_{r-1},\{z\}\right)\right)=+\infty
\end{aligned}
$$

and for any small enough positive $\epsilon$

$$
\begin{aligned}
\liminf _{\beta \rightarrow \infty}-\frac{1}{\beta} \ln P_{\beta}\left(\sum_{n=\theta(G, \tau(D))}^{\tau(D)} 1\left(X_{n}=u\right)<\exp -\beta\left(W_{D \backslash G}(u, u)+\epsilon\right)\right. & \\
/ \pi(X, D, G) & \left.=\left(\{y\}, \pi_{0}, \ldots, \pi_{r-1},\{z\}\right)\right) \geq \epsilon .
\end{aligned}
$$

Proof. We recall that $-W_{D \backslash G}(u, u)=W(\pi(u, D \backslash G))+H(\pi(u, D \backslash G))-$ $W(u)$. These estimations follow from proposition 10.6 .

REMARK. The states of the exit saddle path are not necessarily those in which the exit path spends most of its time. The states in which the exit path spends most of its time are the ground states of the deepest cycles of the exit cycle path.

\section{Chronology of the exit path}

In this section we will be interested in a more refined large deviation upper bound which controls all the arrows of the exit path and the order in which they appear. With the same notation as before, we introduce the cost function

$$
\begin{aligned}
V_{x}(D, G)(X)= & W_{D}\left(x, X_{\theta}\right)+ \\
& \sum_{n=\theta+1}^{\tau(D)}\left(V\left(X_{n-1}, X_{n}\right)+W_{\left\{X_{\theta+1}, \ldots, X_{n-1}\right\}}\left(X_{n-1}, X_{n-1}\right)\right)^{+}
\end{aligned}
$$

with the convention that $W_{\emptyset}(u, u)=0$ in all cases. We consider the sequence of random times $\nu_{0}=0$,

$$
\nu_{k}=\inf \left\{n>\nu_{k-1}: V\left(X_{n-1}, X_{n}\right)+W_{S_{n}}\left(X_{n-1}, X_{n-1}\right)>0\right\} \text {, }
$$

where we have put $S_{n}=\left\{X_{\theta+1}, \ldots, X_{n-1}\right\}$. The cost function defined above is related to the events

$$
\begin{aligned}
\mathcal{E}_{r}\left(y,\left(s_{l}\right),\left(A_{k}\right), z\right)=\left\{X_{\theta}=y, X_{\nu_{k}-1}=s_{2 k-1}, X_{\nu_{k}}=s_{2 k},\right. \\
\left.S_{\nu_{k}}=A_{k}, 1 \leq k \leq r, \tau(D)<\nu_{r+1}, X_{\tau(D)}=z\right\}
\end{aligned}
$$

where the sets $A_{k}$ are subsets of $D \backslash G$ and the points $s_{l}$ are in $D \backslash G$. For each of these events we have the following estimates. 
THEOREM 11.1. There is a positive constant $K_{11}$ such that for any $\beta$

$$
\begin{aligned}
&\left(K_{11}^{-1} a(\beta)^{K_{11}}\right)^{r+1} \exp -\beta V_{x}(\mathcal{E}) \\
& \leq P_{\beta}\left(\mathcal{E}_{r}\left(y,\left(s_{l}\right),\left(A_{k}\right), z\right) / X_{0}=x\right) \leq \\
&\left(K_{11} a(\beta)^{-K_{11}}\right)^{r+1} \exp -\beta V_{x}(\mathcal{E}) .
\end{aligned}
$$

Proof.

$$
\begin{aligned}
P_{\beta}\left(\mathcal{E}_{r}\left(y,\left(s_{l}\right),\left(A_{k}\right), z\right) / X_{0}=\right. & x)=\left(\sum_{m=0}^{\infty} P_{\beta}\left(X_{m}=y, \tau(D)>m / X_{0}=x\right)\right) \\
& \times P_{\beta}\left(\mathcal{E}_{r}\left(y,\left(s_{l}\right),\left(A_{k}\right), z\right), X_{\tau(D \backslash G)} \in D^{c} / X_{0}=y\right) .
\end{aligned}
$$

Moreover

$$
\begin{aligned}
& P_{\beta}\left(\mathcal{E}_{r}\left(y,\left(s_{l}\right),\left(A_{k}\right), z\right), X_{\tau(D \backslash G)} \in D^{c} / X_{0}=y\right)= \\
& \quad P_{\beta}\left(X_{\tau(D \backslash G)}=z, \nu_{r+1}>\tau(D) / X_{\nu_{r}}=s_{2 r}\right) \times \\
& \prod_{k=1}^{r} P_{\beta}\left(X_{\nu_{k}-1}=s_{2 k-1}, X_{\nu_{k}}=s_{2 k}, S_{\nu_{k}}=A_{k} / X_{\nu_{k-1}}=s_{2 k-2}, S_{\nu_{k-1}}=A_{k-1}\right)
\end{aligned}
$$

We have to estimate the term

$$
P_{\beta}\left(X_{\nu_{k}-1}=s_{2 k-1}, X_{\nu_{k}}=s_{2 k}, S_{\nu_{k}}=A_{k} / X_{\nu_{k-1}}=s_{2 k-2}, S_{\nu_{k-1}}=A_{k-1}\right) .
$$

We will deal with the case when $A_{k-1} \neq A_{k}$, which is the most intricate, the other case is left to the reader. Let $\mathcal{I}_{k}$ be the collection of all the sequences $\left(u_{0}, \ldots, u_{m}\right)$ in $\left(A_{k-1}^{c}\right)^{m+1}$ such that

- $u_{0}=s_{2 k-2}$,

- $u_{0}, \ldots, u_{m}$ are distinct points,

- $A_{k-1} \cup\left\{u_{0}, \ldots, u_{m}\right\}=A_{k}$,

- $C_{A_{k-1} \cup\left\{u_{0}, \ldots, u_{l-1}\right\}}\left(u_{l-1}, u_{l}\right)=0$.

If $\mathcal{I}_{k}$ is empty, then the term in formula (11.2) is zero, otherwise it is equal to

$$
\begin{gathered}
\sum_{\left(u_{0}, \ldots, u_{m}\right) \in \mathcal{I}_{k}} \prod_{l=1}^{m} P_{\beta}\left(X_{\alpha_{l}}=u_{l}, \nu_{k}>\alpha_{l} / X_{\alpha_{l-1}}=u_{l-1}\right. \\
\left.S_{u_{l-1}}=A_{k-1} \cup\left\{u_{0}, \ldots, u_{l-2}\right\}\right) \\
\times P_{\beta}\left(X_{\nu_{k}-1}=s_{2 k-1}, X_{\nu_{k}}=s_{2 k}, S_{\nu_{k}}=A_{k} / X_{\alpha_{m}}=u_{m}, S_{\alpha_{m}+1}=A_{k}\right)
\end{gathered}
$$

where $\alpha_{0}=\nu_{k-1}$ and $\alpha_{l}=\inf \left\{n>\alpha_{l-1} / X_{n} \notin S_{n}\right\}$. But

$$
\begin{aligned}
& P_{\beta}\left(X_{\alpha_{l}}=u_{l}, \nu_{k}>\alpha_{l} / \cdots\right)= \\
& P_{\beta}\left(X_{\alpha_{l}}=u_{l} / \cdots\right)-P_{\beta}\left(X_{\alpha_{l}}=u_{l}, \nu_{k} \leq \alpha_{l} / \cdots\right) .
\end{aligned}
$$

In addition, for some positive constants $\gamma, C_{7}, C_{8}$,

$$
\begin{gathered}
P_{\beta}\left(X_{\alpha_{l}}=u_{l}, \nu_{k} \leq \alpha_{l} / \cdots\right) \leq P_{\beta}\left(\nu_{k} \leq \alpha_{l} / \cdots\right) \leq C_{7} a(\beta)^{-C_{8}} \exp (-\gamma \beta), \\
C_{7}^{-1} a(\beta)^{C_{8}} \leq P_{\beta}\left(X_{\alpha_{l}}=u_{l} / \cdots\right) \leq C_{7} a(\beta)^{-C_{8}} .
\end{gathered}
$$


Therefore for $\beta$ large enough and for some positive constants $C_{9}, C_{10}$,

$$
C_{9}^{-1} a(\beta)^{C_{10}} \leq P_{\beta}\left(X_{\alpha_{l}}=u_{l}, \nu_{k}>\alpha_{l} / \cdots\right) \leq C_{9} a(\beta)^{-C_{10}} .
$$

We also have for some positive constants $C_{11}, C_{12}$

$$
\begin{aligned}
& C_{11}^{-1} a(\beta)^{C_{12}} \exp -\beta\left(W_{A_{k}}\left(u_{m}, s_{2 k-1}\right)+V\left(s_{2 k-1}, s_{2 k}\right)\right) \leq \\
& P_{\beta}\left(X_{\nu_{k}-1}=s_{2 k-1}, X_{\nu_{k}}=s_{2 k}, S_{\nu_{k}}=A_{k} / X_{\alpha_{m}}=u_{m}, S_{\alpha_{m}+1}=A_{k}\right) \\
& \leq C_{11} a(\beta)^{-C_{12}} \exp -\beta\left(W_{A_{k}}\left(u_{m}, s_{2 k-1}\right)+V\left(s_{2 k-1}, s_{2 k}\right)\right) .
\end{aligned}
$$

Let us remark eventually that $W_{A_{k}}\left(u_{m}, s_{2 k-1}\right)=W_{A_{k}}\left(s_{2 k-1}, s_{2 k-1}\right)$ because it is possible to go from $u_{m}$ to $s_{2 k-1}$ in $A_{k}$ at null cost. Putting together (11.1), (11.3), (11.4), (11.5) and noticing that $P_{\beta}\left(X_{\tau(D \backslash G)}=z, \nu_{r+1}>\right.$ $\left.\tau(D) / X_{\nu_{r}}=s_{2 r}\right)$ is logarithmically equivalent to zero, we obtain the estimates stated in the theorem.

Theorem 11.2. For any positive $\lambda$ there is a positive constant $K_{12}$ such that, for any $\beta$,

$$
P_{\beta}\left(V_{x}(X) \geq \lambda / X_{0}=x\right) \leq K_{12} a(\beta)^{-K_{12}} \exp -\beta \lambda .
$$

Proof. Let

$$
\alpha=\min \left(\left\{\left(V(x, y)+W_{A}(x, x)\right)^{+}: x, y \in E, A \subset E\right\} \backslash\{0\}\right) .
$$

We write

$$
\begin{gathered}
P\left(\nu_{k}<\tau(D) / X_{0}=x\right)=\sum\left(\left(\sum_{m=0}^{\infty} P_{\beta}\left(X_{m}=y, \tau(D)>m / X_{0}=x\right)\right) \times\right. \\
\left.\prod_{l=1}^{k} P_{\beta}\left(X_{\nu_{l}-1}=s_{2 l-1}, X_{\nu_{l}}=s_{2 l}, S_{\nu_{l}}=A_{l} / X_{\nu_{l-1}}=s_{2 l-2}, S_{\nu_{l-1}}=A_{l-1}\right)\right)
\end{gathered}
$$

where the first summation set is $\left\{y \in G,\left(s_{1}, \ldots, s_{2 k}\right) \in E^{2 k},\left(A_{1}, \ldots, A_{k}\right) \in\right.$ $\left.\mathcal{P}(E)^{k}\right\}$. The same estimates as in theorem 11.1 show that for any $k$ there exist positive constants $C_{13}, C_{14}$ such that

$$
P_{\beta}\left(\nu_{k} \leq \tau(D) / X_{0}=x\right) \leq C_{13} a(\beta)^{-C_{14}} \exp -\beta\left(k \alpha+\min _{y \in G} W_{D}(x, y)\right) .
$$

We choose $k$ such that $k \alpha+\min _{y \in G} W_{D}(x, y)>2 \lambda$ and we write

$$
\begin{aligned}
P_{\beta}\left(V_{x}(X) \geq\right. & \left.\lambda / X_{0}=x\right) \leq \\
& P_{\beta}\left(\nu_{k}<\tau(D) / X_{0}=x\right)+P_{\beta}\left(V_{x}(X) \geq \lambda, \nu_{k} \geq \tau(D) / X_{0}=x\right) .
\end{aligned}
$$

The second term of the righthand side is the probability of a finite union of events of the type described in theorem 11.1; applying the estimates of theorem 11.1 to each of these events yields the desired inequality. 


\section{APPLICATIONS}

In this section, we apply our results to study the way the process falls to the bottom of a cycle or escapes from the bottom of a cycle.

Falling to the воттом. Let $\pi$ be a cycle and $x$ a starting point in $\pi$. We take $D=\pi \backslash F(\pi)$ and $G=\pi(x, D)$ i.e. we want to study the way the chain either leaves $\pi$ or reaches the bottom $F(\pi)$ of the cycle. For any point $s_{0}$ in $\pi(x, D)$ we have $C_{D \backslash\left\{s_{0}\right\}}\left(x, s_{0}\right)=0$ (since $D$ contains the cycle $\pi(x, D)$ ). Theorem 9.1 shows that we must necessarily have (second point of the characterization of the saddle paths of null cost)

$$
V\left(s_{0}, s_{1}\right)=H\left(\pi\left(s_{0}, D\right)\right)+W\left(\pi\left(s_{0}, D\right)\right)-W\left(s_{0}\right) .
$$

Moreover $s_{1}$ does not belong to $G$ and is thus in the boundary of $\pi\left(s_{0}, D\right)=$ $\pi(x, D)$. Since by corollary 7.3

$$
H\left(\pi\left(s_{0}, D\right)\right)+W\left(\pi\left(s_{0}, D\right)\right)-W\left(s_{0}\right) \leq V\left(s_{0}, s_{1}\right)-C_{\pi\left(s_{0}, D\right)}\left(s_{0}, s_{1}\right)
$$

a saddle path of null $V_{x}$-cost has to satisfy $C_{\pi\left(s_{0}, D\right)}\left(s_{0}, s_{1}\right)=0$ so that in fact $s_{1}$ belongs to $\widetilde{B}\left(\pi\left(s_{0}, D\right)\right)$. Necessarily, the point $s_{1}$ belongs to $\pi$. In addition, $C_{D \backslash G}\left(s_{1}, s_{2 r+1}\right)=0$ whence $s_{2 r+1}$ is in the bottom $F(\pi)$ (the exit of $D$ takes place in $F(\pi)$ ). In this situation, the index $r_{*}$ is null, the ascending part of the saddle path is reduced to $\left(s_{0}\right)$.

EscAPING FROM THE BOTTOM. Let $\pi$ be a cycle and $x$ a starting point in the bottom $F(\pi)$. We take $D=\pi$ and $G=F(\pi)$ i.e. we want to study the way the chain leaves $\pi$ after its last visit to $F(\pi)$. For any point $s_{0}$ in $F(\pi)$ we have $\pi\left(s_{0}, D\right)=\pi$ and $C_{D \backslash\left\{s_{0}\right\}}\left(x, s_{0}\right)=0$. By proposition 4.14 we have also $C_{D \backslash\left\{s_{0}\right\}}\left(s_{0}, D^{c}\right)=H(\pi)$. In this situation the indices $r_{*}$ and $r^{*}$ are equal to the length $r$ of the saddle path, the ascending part is $\left(s_{0}, \ldots, s_{2 r}\right)$, the ridge part is empty, the descending part is $\left(s_{2 r+1}\right)$ and $s_{2 r+1}$ belongs to the principal boundary of $\pi$. The global saddles are the optimal saddles exiting from $\pi$ i.e. the saddles $(y, z)$ such that $y \in \pi, z \in \widetilde{B}(\pi)$ and $W(y)+V(y, z)=$ $W(\pi)+H(\pi)$.

Recursive applications of the Result. Knowing that the Markov chain follows a fixed saddle path, we can recursively apply our result to obtain information on the way it enters and exits from the successive cycles of the associated cycle path: for instance, on the way it falls to the bottom or escapes from the bottom of an intermediate cycle.

\section{The Reversible Dynamics of Metropolis}

In this final section, we deal with the reversible case of the Metropolis dynamics. The reversibility of the dynamics induces some reversibility properties for the exit saddle path. This situation has also been studied in Olivieri and Scoppola (1995) and in Schonmann (1992).

Let $U: E \mapsto \mathbb{R}_{+}$be a potential whose global minimum over $E$ is zero. We suppose that

$$
\forall x, y \in E \quad V(x, y)<\infty \quad \Longleftrightarrow \quad V(y, x)<\infty
$$


and that $V(x, y)=(U(y)-U(x))^{+}$whenever $V(x, y)<\infty$. In this situation the virtual energy $W$ coincides with the potential $U$ and each quantity admits a simplified expression with the help of $U$. For instance, for any cycle $\pi, H(\pi)=\min \left\{(U(x)-U(\pi))^{+}: x \in B(\pi)\right\}$.

Proposition 13.1. (reversibility of the saddle path) Let $\mathcal{R}$ be the reversing operator defined on the set of sequences of points of $E$ of odd length by $\mathcal{R}\left(s_{0}, \ldots, s_{2 r+1}\right)=\left(s_{2 r+1}, \ldots, s_{0}\right)$. The operator $\mathcal{R}$ is a one to one map between the saddle paths of $\mathcal{S}(D, G)$ and $\mathcal{S}\left(G^{c}, D^{c}\right)$.

Proof. The map $\mathcal{R}$ is clearly one to one since $\mathcal{R} \circ \mathcal{R}$ is the identity map. That $\mathcal{R}(\mathcal{S}(D, G))=\mathcal{S}\left(G^{c}, D^{c}\right)$ is a consequence of the set equality $D \backslash G=$ $G^{c} \backslash D^{c}$ and of the following facts:

$$
\begin{aligned}
V\left(s_{2 k}, s_{2 k+1}\right)<\infty & \Longleftrightarrow V\left(s_{2 k+1}, s_{2 k}\right)<\infty \\
s_{2 k} \in \pi\left(s_{2 k-1}, D \backslash G\right) & \Longleftrightarrow s_{2 k-1} \in \pi\left(s_{2 k}, G^{c} \backslash D^{c}\right), \\
s_{2 k+1} \in B\left(\pi\left(s_{2 k}, D \backslash G\right)\right) & \Longleftrightarrow s_{2 k} \in B\left(\pi\left(s_{2 k+1}, G^{c} \backslash D^{c}\right)\right) .
\end{aligned}
$$

Lemma 13.2. Let $\left(s_{0}, \ldots, s_{2 r+1}\right)$ be a saddle path. For all $k$ in $\left\{1, \ldots, r_{*}\right\}$ we have

$$
\begin{aligned}
U\left(s_{2 k}\right)+V\left(s_{2 k}, s_{2 k+1}\right)-H\left(\pi\left(s_{2 k}, D \backslash G\right)\right)-U\left(\pi\left(s_{2 k}, D \backslash G\right)\right) \\
\geq \max \left(U\left(s_{2 k}\right), U\left(s_{2 k+1}\right)\right)-\max \left(U\left(s_{2 k-2}\right), U\left(s_{2 k-1}\right)\right) .
\end{aligned}
$$

Proof. Since $s_{2 k-2}$ does not belong to $\pi\left(s_{2 k-1}, D \backslash G\right)$ (which coincides with $\pi\left(s_{2 k}, D \backslash G\right)$ ) and $V\left(s_{2 k-2}, s_{2 k-1}\right)$ is finite (which implies by reversibility that $V\left(s_{2 k-1}, s_{2 k-2}\right)$ is also finite), then $s_{2 k-2}$ belongs to the boundary of the cycle $\pi\left(s_{2 k}, D \backslash G\right)$. Either $\pi\left(s_{2 k}, D \backslash G\right)$ is reduced to $\left\{s_{2 k}\right\}$ and $H\left(\pi\left(s_{2 k}, D \backslash G\right)\right)=0$, in which case the inequality of the lemma is satisfied, or $H\left(\pi\left(s_{2 k}, D \backslash G\right)\right)>0$; in this last case, we have

$$
U\left(s_{2 k-2}\right) \geq H\left(\pi\left(s_{2 k}, D \backslash G\right)\right)+U\left(\pi\left(s_{2 k}, D \backslash G\right)\right)
$$

which gives the desired inequality.

Theorem 13.3. (reversibility of the ascending part) Let $\left(s_{0}, \ldots, s_{2 r+1}\right)$ be a saddle path of $\mathcal{S}(D, G)$ of null $V_{s_{0}}(D, G)$-cost.

For any $k$ in $\left\{1, \ldots, r_{*}\right\}$, the point $s_{2 k-2}$ belongs to the principal boundary of $\pi\left(s_{2 k-1}, D \backslash G\right)$. Equivalently, the ascending part $\left(s_{0}, \ldots, s_{2 r_{*}}\right)$ of the saddle path $\left(s_{0}, \ldots, s_{2 r+1}\right)$ is the descending part of a saddle path of $\mathcal{S}\left(G^{c}, \pi\left(s_{0}, D\right)^{c}\right)$ of null $V_{s_{2 r_{*}+1}}\left(G^{c}, \pi\left(s_{0}, D\right)^{c}\right)$-cost.

Remark. For $s \in \pi\left(s_{0}, D\right)$ we have $\pi(s, D \backslash G)=\pi\left(s, \pi\left(s_{0}, D\right) \backslash G\right)=$ $\pi\left(s, G^{c} \backslash \pi\left(s_{0}, D\right)^{c}\right)$.

Proof. Let $\left(s_{0}, \ldots, s_{2 r+1}\right)$ be a saddle path of null $V_{s_{0}}-$ cost. We have by lemma 13.2

$$
\begin{array}{r}
V\left(s_{0}, s_{1}\right)+ \\
\sum_{1 \leq k \leq r_{*}}\left(U\left(s_{2 k}\right)+V\left(s_{2 k}, s_{2 k+1}\right)-H\left(\pi\left(s_{2 k}, D \backslash G\right)\right)-U\left(\pi\left(s_{2 k}, D \backslash G\right)\right)\right) \\
\geq V\left(s_{0}, s_{1}\right)+\sum_{1 \leq k \leq r_{*}} \max \left(U\left(s_{2 k}\right), U\left(s_{2 k+1}\right)\right)-\max \left(U\left(s_{2 k-2}\right), U\left(s_{2 k-1}\right)\right) \\
\geq U\left(s_{2 r_{*}+1}\right)-U\left(s_{0}\right) .
\end{array}
$$


Now $s_{2 r_{*}+1}$ belongs to the principal boundary of $\pi\left(s_{0}, D\right)$ whence

$$
U\left(s_{2 r_{*}+1}\right)-U\left(s_{0}\right)=U\left(\pi\left(s_{0}, D\right)\right)+H\left(\pi\left(s_{0}, D\right)\right)-U\left(s_{0}\right)
$$

which is also the value of the initial sum in the above formula (a necessary condition for $\left(s_{0}, \ldots, s_{2 r+1}\right)$ to be a saddle path of null $V_{s_{0}}$-cost, see the second condition given in theorem 9.1). It follows that all the intermediate inequalities (given by lemma 13.2) are in fact equalities i.e. for all $k$ in $\left\{1, \ldots, r_{*}\right\}$

$$
U\left(\pi\left(s_{2 k-1}, D \backslash G\right)\right)+H\left(\pi\left(s_{2 k-1}, D \backslash G\right)\right)=\max \left(U\left(s_{2 k-2}\right), U\left(s_{2 k-1}\right)\right) .
$$

This equality is equivalent to the fact that $s_{2 k-2}$ belongs to the principal boundary of $\pi\left(s_{2 k-1}, D \backslash G\right)$.

THEOREM 13.4. (global reversibility of saddle paths of null cost between two cycles) Let $\pi_{1}$ and $\pi_{2}$ be two disjoint cycles of $E$ and set $G=\pi_{1}$, $D=\pi_{2}^{c}$. The reversing operator $\mathcal{R}$ maps the saddle paths of null cost of $\mathcal{S}(D, G)$ onto the saddle paths of null cost of $\mathcal{S}\left(G^{c}, D^{c}\right)$; it leaves the ridge part invariant and exchanges the ascending and the descending part. More precisely, let $\left(s_{0}, \ldots, s_{2 r+1}\right)$ be a saddle path of $\mathcal{S}(D, G)$ of null $V_{s_{0}}-$ cost. Then $\mathcal{R}\left(s_{0}, \ldots, s_{2 r+1}\right)$ is a saddle path of $\mathcal{S}\left(G^{c}, D^{c}\right)$ of null $V_{s_{2 r+1}}-$ cost. Its ascending part is $\left(s_{2 r+1}, \ldots, s_{2 r^{*}+1}\right)$, its ridge part is $\left(s_{2 r^{*}}, \ldots, s_{2 r_{*}+1}\right)$ and its descending part $\left(s_{2 r_{*}}, \ldots, s_{0}\right)$.

Proof. Let $\left(s_{0}, \ldots, s_{2 r+1}\right)$ be a saddle path of null $V_{s_{0}}-$ cost. Proposition 13.1 shows that $\mathcal{R}\left(s_{0}, \ldots, s_{2 r+1}\right)$ belongs to $\mathcal{S}\left(G^{c}, D^{c}\right)$. We have

$$
\begin{array}{r}
\sum_{1 \leq k \leq r}\left(U\left(s_{2 k}\right)+V\left(s_{2 k}, s_{2 k+1}\right)-H\left(\pi\left(s_{2 k}, D \backslash G\right)\right)-U\left(\pi\left(s_{2 k}, D \backslash G\right)\right)\right)= \\
\sum_{1 \leq k \leq r}\left(\max \left(U\left(s_{2 k-1}\right), U\left(s_{2 k-2}\right)\right)-H\left(\pi\left(s_{2 k-1}, D \backslash G\right)\right)-U\left(\pi\left(s_{2 k-1}, D \backslash G\right)\right)\right) \\
-\max \left(U\left(s_{1}\right), U\left(s_{0}\right)\right)+\max \left(U\left(s_{2 r+1}\right), U\left(s_{2 r}\right)\right)
\end{array}
$$

so that

$$
\begin{gathered}
V_{s_{0}}\left(s_{0}, \ldots, s_{2 r+1}\right)-V_{s_{2 r+1}}\left(\mathcal{R}\left(s_{0}, \ldots, s_{2 r+1}\right)\right)=V\left(s_{0}, s_{1}\right)-V\left(s_{2 r+1}, s_{2 r}\right) \\
-\max \left(U\left(s_{1}\right), U\left(s_{0}\right)\right)+\max \left(U\left(s_{2 r+1}\right), U\left(s_{2 r}\right)\right)+W_{D}\left(s_{0}, s_{0}\right) \\
-W_{G^{c}}\left(s_{2 r+1}, s_{2 r+1}\right) \\
=U\left(\pi\left(s_{2 r+1}, G^{c}\right)\right)+H\left(\pi\left(s_{2 r+1}, G^{c}\right)\right)-U\left(\pi\left(s_{0}, D\right)\right)-H\left(\pi\left(s_{0}, D\right)\right)
\end{gathered}
$$

(where we have used lemma 4.7 and proposition 4.14 to compute $W_{D}$ and $\left.W_{G^{c}}\right)$. However the cycles $\pi\left(s_{0}, D\right)$ and $\pi\left(s_{2 r+1}, G^{c}\right)$ do not depend on the points $s_{0}, s_{2 r+1}$ in $\pi_{1} \times \pi_{2}$ : these cycles are two disjoint cycles which are maximal proper subcycles of the smallest cycle $\pi$ containing both $\pi_{1}$ and $\pi_{2}$. As a consequence, the quantities $U\left(\pi\left(s_{0}, D\right)\right)+H\left(\pi\left(s_{0}, D\right)\right)$ and $U\left(\pi\left(s_{2 r+1}, G^{c}\right)\right)+H\left(\pi\left(s_{2 r+1}, G^{c}\right)\right)$ are equal to the level of $\pi$ whence

$$
V_{s_{0}}\left(s_{0}, \ldots, s_{2 r+1}\right)=V_{s_{2 r+1}}\left(\mathcal{R}\left(s_{0}, \ldots, s_{2 r+1}\right)\right) .
$$


Thus $\mathcal{R}\left(s_{0}, \ldots, s_{2 r+1}\right)$ has a null $V_{s_{2 r+1}}-$ cost. The assertion concerning the three parts of $\mathcal{R}\left(s_{0}, \ldots, s_{2 r+1}\right)$ is a straightforward consequence of the definition (proposition 9.3).

Corollary 13.5. Let $\pi_{1}$ and $\pi_{2}$ be two disjoint cycles of $E$. The set of global saddles (respectively the set of global saddle points) between $\pi_{1}$ and $\pi_{2}$ coincides with the set of global saddles (resp. the set of global saddle points) between $\pi_{2}$ and $\pi_{1}$.

\section{APPENDIX}

In this appendix, we will state the counterpart of the main results of the paper in the non homogeneous case. We will rely on Trouvés proofs, therefore we will assume that $a(\beta)=a=$ constant is independent of $\beta$.

We consider a family of time inhomogeneous Markov chains $\left(P_{(\beta .)}\right)$ on $E^{\mathbb{N}}$, indexed by non decreasing sequences $\left(\beta_{k}\right)_{k \in \mathbb{N}}$ of positive real inverse temperatures. We use $\left(X_{n}\right)_{n \in \mathbb{N}}$ to denote the canonical process on $E^{\mathbb{N}}$ and assume that for some positive constant $a$ and some family $\left(p_{\beta}\right)_{\beta \in \mathbb{R}_{+}}$ of Markov matrices we have for any $x, y \in E$

$$
a \leq p_{\beta}(x, y) \exp \beta V(x, y) \leq a^{-1}, \quad \beta \in \mathbb{R}_{+},
$$

and

$$
P_{(\beta .)}\left(X_{n}=y / X_{n-1}=x\right)=p_{\beta_{n}}(x, y) .
$$

Theorem 14.1. (corresponding to theorem 5.2.) There is a positive constant $K$ depending only on $|E|$ and $a$, such that for any non decreasing sequence $(\beta$.), any subsets $D$ and $G, G \subset D$,

$$
\begin{aligned}
& P_{(\beta .)}\left(\pi(D, G)=\left(\{y\}, \pi_{0}, \ldots, \pi_{r-1},\{z\}\right), \tau(D)>n\right. \\
& \left.\qquad \theta(G, \tau(D))=m, X_{\theta(G, \tau(D))}=y\right) \\
& \leq K \exp -\beta_{m+1}\left(V\left(y, \pi_{0}\right)+\sum_{k=1}^{r} C_{\pi_{k-1}}\left(\pi_{k-1}, \pi_{k}\right)\right) \\
& \quad \times \prod_{l=m+1}^{n}\left(1-K^{-1} \exp \left(-\beta_{l} \max _{k=0, \ldots, r-1} H\left(\pi_{k}\right)\right)\right)
\end{aligned}
$$

and

$$
\begin{gathered}
\sum_{l=m+1}^{n} \exp \beta_{l}\left(V\left(y, \pi_{0}\right)+\sum_{k=1}^{r} C_{\pi_{k-1}}\left(\pi_{k-1}, \pi_{k}\right)\right) \\
\times P_{(\beta .)}\left(\pi(D, G)=\left(\{y\}, \pi_{0}, \ldots, \pi_{r-1},\{z\}\right), \tau(D)=l\right. \\
\quad / \theta(G, \tau(D))=m, X_{\theta(G, \tau(D))=y)} \\
\geq K^{-1}-\prod_{l=m+1}^{r}\left(1-K^{-1} \exp \left(-\beta_{l} \max _{k=0, \ldots, r-1} H\left(\pi_{k}\right)\right)\right) .
\end{gathered}
$$


Consequently

$$
\begin{aligned}
P_{(\beta .)}\left(\pi(D, G)=\left(\{y\}, \pi_{0}, \ldots, \pi_{r-1},\{z\}\right),\right. & \tau(D) \leq n \\
& \left./ \theta(G, \tau(D))=m, X_{\theta(G, \tau(D))}=y\right) \\
\geq K^{-1} \exp -\beta_{n}\left(V\left(y, \pi_{0}\right)+\sum_{k=1}^{r} C_{\pi_{k-1}}\left(\pi_{k-1}, \pi_{k}\right)\right) & \\
& \quad \times\left(1-K \prod_{l=m+1}^{n}\left(1-K^{-1} \exp \left(-\beta_{l} \max _{k=0, \ldots, r-1} H\left(\pi_{k}\right)\right)\right)\right) .
\end{aligned}
$$

Proof. With the notations of Catoni (1992) and Trouvé (1993) (English translation Trouvé (1996b)),

$$
\begin{array}{r}
P_{(\beta .)}\left(\pi(D, G)=\left(\{y\}, \pi_{0}, \ldots, \pi_{r-1},\{z\}\right), \tau(D)=n\right. \\
\left./ \theta(G, \tau(D))=m, X_{\theta}=y\right)= \\
{\left[M\left(\emptyset, \pi_{0}\right) M\left(\pi_{0}, \pi_{1}\right) \cdots M\left(\pi_{r-1},\{z\}\right)\right]_{y, m}^{z, n},}
\end{array}
$$

and the result follows by applying theorem $1.43 \mathcal{H}_{2}$ and $\mathcal{H}_{3}$ of Trouvé (1996a) (th. 4.5 of Trouvé (1996b)) to each kernel $M\left(\pi_{k-1}, \pi_{k}\right)$ and using composition lemma 1.40 (lemma 4.2 of Trouvé (1996b)) to combine these estimates.

Theorem 14.2. (corresponding to theorem 6.2) There is a positive constant $K$, depending only on $|E|$ and $a$, such that for any non increasing sequence $(\beta$.), any subsets $D$ and $G, G \subset D$,

$$
\begin{aligned}
P_{(\beta .)}\left(\bar{\pi}(D, G)=\left(\{y\}, \bar{\pi}_{0}, \ldots, \bar{\pi}_{r-1},\{z\}\right), \tau(D)>n\right. \\
\left.\qquad \theta(G, \tau(D))=m, X_{\theta(G, \tau(D))}=y\right) \\
\leq K \exp -\beta_{m+1}\left(V\left(y, \bar{\pi}_{0}\right)+\sum_{k=1}^{r} C_{\bar{\pi}_{k-1}}\left(\bar{\pi}_{k-1}, \bar{\pi}_{k}\right)\right) \\
\quad \times \prod_{l=m+1}^{n}\left(1-K^{-1} \exp \left(-\beta_{l} \max _{k=0, \ldots, r-1} H\left(\bar{\pi}_{k}\right)\right)\right) .
\end{aligned}
$$

On the other hand

$$
\begin{aligned}
& \sum_{l=m+1}^{n} \exp \beta_{l}\left(V\left(y, \bar{\pi}_{0}\right)+\sum_{k=1}^{r} C_{\bar{\pi}_{k-1}}\left(\bar{\pi}_{k-1}, \bar{\pi}_{k}\right)\right) \\
& \times P_{(\beta .)}\left(\bar{\pi}(D, G)=\left(\{y\}, \bar{\pi}_{0}, \ldots, \bar{\pi}_{r-1},\{z\}\right), \tau(D)=l\right. \\
& \left.\quad / \theta(G, \tau(D))=m, X_{\theta(G, \tau(D))}=y\right) \\
& \geq K^{-1}-\prod_{l=m+1}^{n}\left(1-K^{-1} \exp \left(-\beta_{l} \max _{k=0, \ldots, r-1} H\left(\bar{\pi}_{k}\right)\right)\right) .
\end{aligned}
$$

Proof. We have

$$
\begin{array}{r}
P_{(\beta .)}\left(\bar{\pi}(D, G)=\left(\{y\}, \bar{\pi}_{0}, \ldots, \bar{\pi}_{r-1},\{z\}\right), \tau(D)=n / \theta=m, X_{\theta}=y\right) \\
=\left[M\left(\emptyset, \bar{\pi}_{0}\right) M\left(\bar{\pi}_{0}, \bar{\pi}_{1}\right) \cdots M\left(\bar{\pi}_{r-1},\{z\}\right)\right]_{y, m}^{z, n},
\end{array}
$$


with

$$
M\left(\bar{\pi}_{k-1}, \bar{\pi}_{k}\right)=\sum_{G \in \mathcal{M}\left(\bar{\pi}_{k-1}\right)} M\left(\bar{\pi}_{k-1}, G\right) M\left(G, \bar{\pi}_{k}\right)
$$

and the result comes from theorem $1.43 \mathcal{H}_{3}$ and $\mathcal{H}_{4}$ of Trouve (1996a) (theorem 4.5 of Trouvé (1996b)), for the upper bound, and from theorem 1.43 $\mathcal{H}_{2}$ for the lower bound.

As for theorem 7.4, what Trouvé (1996a) allows to do most straightforwardly is to compute the probability of the odd points of the saddle path.

ThEOREM 14.3. (corresponding to theorem 7.4) There is a positive constant $K$, depending on $|E|$ and $a$ only, such that for any non increasing sequence $(\beta$.), any subsets $D$ and $G, G \subset D$,

$$
\begin{gathered}
P_{(\beta .)}\left(s_{1}=z_{0}, \ldots, s_{2 k+1}=z_{k}, \ldots, s_{2 r+1}=z_{r}, \tau(D)>n\right. \\
\left./ \theta(G, \tau(D))=m, X_{\theta}=y\right) \\
\leq K \exp -\beta_{m+1}\left(V\left(y, z_{0}\right)+\sum_{k=1}^{r} C_{\pi_{k-1}}\left(\pi_{k-1}, z_{k}\right)\right) \\
\times \prod_{l=m+1}^{n}\left(1-K^{-1} \exp \left(-\beta_{l} \max _{0 \leq k<r} H\left(\pi_{k}\right)\right)\right),
\end{gathered}
$$

and

$$
\begin{aligned}
& \sum_{l=m+1}^{n} \exp \beta_{l}\left(V\left(y, z_{0}\right)+\sum_{k=1}^{r} C_{\pi_{k-1}}\left(\pi_{k-1}, z_{k}\right)\right) \\
& \times P_{(\beta .)}\left(s_{1}=z_{0}, \ldots, s_{2 r+1}=z_{r}, \tau(D)=l / \theta(G, \tau(D))=m, X_{\theta}=y\right) \\
& \quad \geq K^{-1}-\prod_{l=m+1}^{r}\left(1-K^{-1} \exp \left(-\beta_{l} \max _{0 \leq k<r} H\left(\pi_{k}\right)\right)\right) .
\end{aligned}
$$

Proof.

$$
\begin{aligned}
P_{(\beta .)}\left(s_{1}=z_{0}, \ldots, s_{2 k+1}=z_{k}, \ldots, \tau(D)=n / \theta(G, \tau(D))=m, X_{\theta}=y\right) \\
=\left[M\left(\emptyset,\left\{z_{0}\right\}\right) M\left(\pi_{0},\left\{z_{1}\right\}\right) M\left(\pi_{1},\left\{z_{2}\right\}\right) \cdots M\left(\pi_{r-1},\left\{z_{r}\right\}\right)\right]_{y, m}^{z_{r}, n},
\end{aligned}
$$

and the result follows from theorem 1.43 of Trouvé (1996a) (4.5 of Trouvé (1996b)).

Remark. Getting estimates for the whole saddle path from this theorem is straightforward, since estimates for the whole saddle path (including its even points) are nothing but estimates for the odd points of the saddle path of the chain $\left(X_{n-1}, X_{n}\right)$ with state space $\{(x, y) \in E / V(x, y)<+\infty\}$. We leave the details to the reader. We let also the reader generalize the results of section 11 on the chronology of the exit path, applying Trouvé (1996a) to the chain $\left(X_{n-1}, X_{n}\right)$. 
Theorem 14.4. (corresponding to theorem 8.2) There is a positive constant $K$, depending only on $|E|$ and $a$, such that for any non increasing sequence $(\beta$.), any subsets $D$ and $G, G \subset D$,

$$
\begin{gathered}
P_{(\beta .)}\left(\bar{s}_{1}=z_{0}, \ldots, \bar{s}_{2 r+1}=z_{r}, \tau(D)>n / \theta(G, \tau(D))=m, X_{\theta}=y\right) \\
\leq K \exp -\beta_{m+1}\left(V\left(y, z_{0}\right)+\sum_{k=1}^{r} C_{\bar{\pi}_{k-1}}\left(\bar{\pi}_{k-1}, z_{k}\right)\right) \\
\times \prod_{l=m+1}^{n}\left(1-K^{-1} \exp -\beta_{l} \max _{0 \leq k<r} H\left(\bar{\pi}_{k}\right)\right),
\end{gathered}
$$

and

$$
\begin{aligned}
& \sum_{l=m+1}^{n} \exp \beta_{l}\left(V\left(y, z_{0}\right)+\sum_{k=1}^{r} C_{\bar{\pi}_{k-1}}\left(\bar{\pi}_{k-1}, z_{k}\right)\right) \\
& \times P_{\left(\beta_{.}\right)}\left(\bar{s}_{1}=z_{0}, \ldots, \bar{s}_{2 r+1}=z_{r}, \tau(D)=n / \theta(G, \tau)=m, X_{\theta}=y\right) \\
& \geq K^{-1}-\prod_{l=m+1}^{n}\left(1-K^{-1} \exp -\beta_{l} \max _{0 \leq k<r} H\left(\bar{\pi}_{k}\right)\right) .
\end{aligned}
$$

Proof.

$$
\begin{aligned}
P_{(\beta .)}\left(\bar{s}_{1}=z_{0}, \ldots, \bar{s}_{2 r+1}=\right. & \left.z_{r}, \tau(D)=n / \theta(G, \tau)=m, X_{\theta}=y\right) \\
& =\left[M\left(\emptyset,\left\{z_{0}\right\}\right) M\left(\bar{\pi}_{0},\left\{z_{1}\right\}\right) \cdots M\left(\bar{\pi}_{r-1},\left\{z_{r}\right\}\right)\right]_{y, m}^{z_{r}, n},
\end{aligned}
$$

and

$$
M\left(\bar{\pi}_{k-1},\left\{z_{k}\right\}\right)=\sum_{G \in \mathcal{M}\left(\bar{\pi}_{k-1}\right)} M\left(\bar{\pi}_{k-1}, G\right) M\left(G,\left\{z_{k}\right\}\right) .
$$

Remark. It is also possible to derive from Trouvé (1996a) estimates for the series

$$
\sum_{l} P_{(\beta .)}\left(X_{l}=y, \tau(D, m)>l / X_{m}=x\right)
$$

starting as in the remark following lemma 4.7 from the following inequalities:

$$
\begin{aligned}
& \sum_{l=n+1}^{N} P_{(\beta .)}\left(X_{l}=y, \tau(D, m)>l / X_{m}=x\right) \\
& \times P_{(\beta .)}\left(X_{\tau(D \backslash\{y\}, l+1)} \notin D / X_{l}=y\right) \\
& \leq \sum_{l=m}^{+\infty} P_{(\beta .)}\left(X_{\tau(D \backslash\{y\}, m)}=y, \tau(D \backslash\{y\}, m)=l / X_{m}=x\right) \\
& \times P_{(\beta .)}\left(\tau(D, l)>n / X_{l}=y\right),
\end{aligned}
$$


and

$$
\begin{aligned}
\sum_{l=m}^{n} P_{(\beta .)}\left(X_{l}=y, \tau\right. & \left.(D, m)>l / X_{m}=x\right) \\
& \quad \times P_{(\beta .)}\left(X_{\tau(D \backslash\{y\}, l+1)} \notin D / X_{l}=y\right) \\
\geq & \sum_{l=m}^{n} P_{(\beta .)}\left(X_{\tau(D \backslash\{y\}, m)}=y, \tau(D \backslash\{y\}, m)=l / X_{m}=x\right) \\
& \times P_{(\beta .)}\left(\tau(D, l) \leq n+1 / X_{l}=y\right) .
\end{aligned}
$$

In other words, if the last visit to $y$ is posterior to time $n$, then the exit from $D$ is posterior to $n$, and occurred after $y$ has been visited. On the other hand, if the chain leaves $D$ before time $n+1$, and has visited $y$ in the meantime, then its last visit to $y$ occurred before time $n$.

Applying the first inequality to the inverse temperature sequence $\tilde{\beta}_{l}=$ $\beta_{l} \wedge \beta_{N}$, and the second one directly to the sequence $(\beta$.), and using theorem 1.43 of Trouvé (1996a), we get the following theorem.

Theorem 14.5. There is a positive constant $K$, depending only on $|E|$ and $a$, such that for any non increasing sequence $(\beta$.), any subset $D$, any points $x, y \in D$, any times $m \leq n<N$,

$$
\begin{aligned}
\sum_{l=n+1}^{N} P_{(\beta .)}\left(X_{l}=\right. & \left.y, \tau(D, m)>l / X_{m}=x\right) \\
\leq K \exp \left(\beta_{N} W_{D}(y, y)-\right. & \left.\beta_{m+1} C_{D \backslash\{y\}}(x, y)\right) \\
& \times \prod_{l=m+1}^{n}\left(1-K^{-1} \exp -\beta_{l} H(D)\right),
\end{aligned}
$$

and

$$
\begin{aligned}
& \sum_{l=m}^{n} P_{(\beta .)}\left(X_{l}=y, \tau(D, m)>l /\right.\left.X_{m}=x\right) \\
& \geq \exp \left(\beta_{m+1} W_{D}(y, y)-\beta_{n} C_{D \backslash\{y\}}(x, y)\right) \\
& \quad \times\left(K^{-1}-\prod_{l=m+1}^{n}\left(1-K^{-1} \exp -\beta_{l} H(D)\right)\right) .
\end{aligned}
$$

Therefore the whole of theorems 5.2, 6.2, 7.4 and 8.2 can be deduced from Trouvé (1996a), or from its English translation Trouvé (1996b), in the case $a(\beta)=a=$ constant, using only simple identities based on the Markov property.

To prove the case when $a(\beta)$ depends on $\beta$ from Trouvé (1996a), one has to check moreover that the constant $K$ in the preceding theorems of this appendix can be chosen to be polynomial in $a$, which amounts to a careful inspection of the proofs of Trouve (1996a). Thus, the whole of the present paper can be derived by an induction proof, also valid in the non homogeneous case, without any use of Freidlin and Wentzell identities based on graph summations. 


\section{BIBLIOGRAPHY}

Alonso, L. and CERF, R. (1996), The three dimensional polyominoes of minimal area, Electronic Journal of Combinatorics $\mathbf{3}$ \#R27.

Ben Arous, G. and CERF, R. (1996), Metastability of the three dimensional Ising model on a torus at very low temperatures, Electronic Journal of Probability 1 1-55.

Cassandro, M., Galves, A., Olivieri, E. and Vares, M.E. (1984), Metastable behaviour of stochastic dynamics: a pathwise approach, Jour. Stat. Phys. $\mathbf{3 5}$ nos. 5/6 603-634.

Catoni, O. (1988), Grandes déviations et décroissance de la température dans les algorithmes de recuit, C.R. Acad. Sci. Paris Sér. $1 \quad \mathbf{3 0 7}$ 535-538.

Catoni, O. (1990), Large deviations for annealing, PhD Thesis, University Paris XI.

CAtoni, O. (1991a), Sharp large deviation estimates for simulated annealing algorithms, Ann. Inst. Henri Poincaré Vol. 27 no. 3 291-383.

Catoni, O. (1991b), Applications of Sharp Large Deviation Estimates to Optimal Cooling Schedules, Ann. Inst. Henri Poincaré 27 463-518.

CAtoni, O. (1992), Rough large deviation estimates for simulated annealing. Application to exponential schedules, Annals of Probab. $201109-1146$.

Catoni, O. (1994), The energy transformation method for the Metropolis algorithm compared with simulated annealing, Probab. Theory and Rel. Fields (to appear).

CAtoni, O. (1995), Algorithmes de recuit simulé et chaînes de Markov à transitions rares. Notes de cours de DEA, English translation: Simulated Annealing Algorithms and Markov Chains with Rare Transitions (1996), Université Paris 11, lecture notes, DEA Stochastic Models and Statistics.

CAtoni, O. and Cot, C. (1996), Rate of Convergence of Generalized Simulated Annealing with Piecewise Constant Triangular Cooling Schedules, preprint Rapport de Recherche du L.M.E.N.S. .

CERF, R. (1993), Asymptotic convergence of genetic algorithms, preprint.

CERF, R. (1994), Une théorie asymptotique des algorithmes génétiques, PhD Thesis, Université Montpellier II.

CERF, R. (1996a), An asymptotic theory for genetic algorithms, Artificial Evolution, Lecture Notes in Computer Science 1063, Springer-Verlag, 37-53.

CERF, R. (1996b), The dynamics of mutation-selection algorithms with large population sizes, Ann. Inst. Henri Poincaré 32 no. 4 455-508.

CERF, R. (1996c), A new genetic algorithm, Annals Applied Probab. Vol. 6 no. 3 778817.

Chen, D., Feng, J. and QIAn, M. (1995), The Metastability of Exponentially Perturbed Markov Chains, Chinese Science A 25(6) 590-595.

Chiang, T.S. and Chow, Y. (1989), A Limit Theorem for a Class of Inhomogeneous Markov Processes, Annals of Probab. 17 no 4 1483-1502.

Chinng, T.S. and Chow, Y. (1995), On the Exit Problem from a Cycle of Simulated Annealing Processes, Tech. Rept. Inst. of Math. Academia Sinica.

Diaconis, P. and STRoock, D. (1991), Geometric bounds for eigenvalues of Markov chains, Annals Applied Probab. Vol. 1 no. 1 36-61.

Deuschel, J.D. and MazzA, C. (1994), $L^{2}$ convergence of time nonhomogeneous Markov processes: I. Spectral Estimates, Annals Applied Probab. Vol. 4 no. $41012-$ 1056.

Freidlin, M.I. and Wentzell, A.D. (1984), Random perturbations of dynamical systems, Springer-Verlag, New York.

Götze, F. (1991), Rate of Convergence of Simulated Annealing Processes, preprint.

Holley, R. and Stroock, D.W. (1988), Simulated Annealing via Sobolev Inequalities, Commun. Math. Phys. Vol. 115 553-569.

Holley, R., Kusuoka, S. and Stroock, D. (1989), Asymptotics of the Spectral Gap with Applications to the Theory of Simulated Annealing, J. Funct. Anal. $\mathbf{8 3}$ $333-347$.

Hwang, C.R. and Sheu, S.J. (1986), Large Time Behaviors of Perturbed Diffusion Markov Processes with Applications III Simulated Annealing, preprint, cited in Chiang and Chow (1989). 
Hwang, C.R. and SHEu, S.J. (1992), Singular perturbed Markov chains and exact behaviour of simulated annealing processes, J. Theoret. Prob. Vol. 5 no. 2 223-249.

Kotecky, R. and Olivieri, E. (1993), Droplet dynamics for asymmetric Ising model, Jour. Stat. Phys. 70 nos. 5/6 1121-1148.

Kotecky, R. and Olivieri, E. (1994), Shapes of growing droplets - a model of escape from a metastable phase, Jour. Stat. Phys. 75 nos 3/4 409-506.

Miclo, L. (1991), Evolution de l'énergie libre. Application à l'étude de la convergence des algorithmes de recuit simulé, Thèse, Université Paris XI-Orsay.

Miclo, L. (1995), Sur les temps d'occupations des processus de Markov finis inhomogènes à basse température, preprint, submitted to Stochastics and Stochastics Reports.

Miclo, L. (1996), Sur les problèmes de sortie discrets inhomogènes, Annals Applied Probab. (to appear).

Neves, E.J. and Schonmann, R.H. (1991), Critical droplets and metastability for a Glauber dynamics at very low temperatures, Commun. Math. Phys. $137209-$ 230.

Neves, E.J. and Schonmann, R.H. (1992), Behaviour of droplets for a class of Glauber dynamics at very low temperatures, Prob. Th. Related Fields 91 331-354.

OLIVIERI, E. and SCOPPOLA, E. (1995), Markov chains with exponentially small transition probabilities: first exit problem from a general domain -I. The reversible case, Journ. Stat. Phys. 79 613-647.

OLIVIERI, E. and SCOPPOLA, E. (1996), Markov chains with exponentially small transition probabilities: first exit problem from a general domain -II. The general case, Journ. Stat. Phys. (to appear).

SCHONMANN, R.H. (1992), The pattern of escape from metastability of a stochastic Ising model, Commun. Math. Phys 147 231-240.

SCOPPOLA, E. (1993), Renormalization group for Markov chains: a general procedure based on renormalization group ideas, Jour. Stat. Phys. 73 nos. 1/2 83-121.

Trouvé, A. (1992), Convergence optimale pour les algorithmes de recuits généralisés, $C$. R. Acad. Sci. Paris t.315 Série I 1197-1202.

Trouvé, A. (January 1993), Parallélisation massive du recuit simulé, PhD Thesis, University Paris XI.

Trouvé, A. (1996a), Cycle decompositions and simulated annealing, SIAM J. Control Optimization 34 no. 3 966-986.

Trouvé, A. (1996b), Rough large deviation estimates for the optimal convergence speed exponent of generalized simulated annealing algorithms, Ann. Inst. Henri Poincaré 32 no. 3 299-348.

Tsitsiklis, J.N. (1989), Markov Chains with Rare Transitions and Simulated Annealing, Math. Oper. Res. 14 70-90.

O. Catoni, Diam, Laboratoire de Mathématioues de l'Ecole Normale SupéRIEURE, UA 762 DU CNRS, 45 RUE D'Ulm, 75230 Paris Cedex 05, France. Email: Olivier.Catonidens.fr.

R. Cerf, Université Paris Sud, Mathématique, Bâtiment 425, Modf́lisation stochastique et statistique, URA D 0743 du CNRS, 91405 Orsay Cedex, France. EMAIL: Raphael.Cerfomath.u-psud.fr. 